\title{
Numerical Investigation of Hydrodynamic Instabilities in Singing Risers
}

\author{
J. Brac, L.-M. Berthe and F. Pradel \\ IFP Energies nouvelles, 1-4 avenue de Bois-Préau, 92852 Rueil-Malmaison Cedex - France \\ e-mail: jean.brac@ifpen.fr - francis.pradel@ifpen.fr
}

\begin{abstract}
Résumé - Investigation numérique des instabilités hydrodynamiques de risers chantants Le transport offshore de gaz utilise souvent des conduites flexibles. Elles sont fabriquées en enroulant en hélice un ruban d'acier; au contact bord à bord des tours d'hélice, une cavité apparaît. Le gaz s'écoule en conduite et rencontre cette cavité à intervalles réguliers, toujours avec la même forme. Dans certaines conditions d'usage, des vibrations acoustiques de grandes amplitudes apparaissent. En effet, devant chaque cavité, il y a une couche de cisaillement où une instabilité de type Kelvin-Helmholtz apparaît. L'écoulement rencontre un très grand nombre de fois la cavité sur son chemin et une question se pose : existe-t-il un écoulement stabilisé ?

Les résultats des simulations des grandes échelles de la turbulence sont présentés en référence mais elles sont extrêmement coûteuses. Cela justifie le développement de solutions numériques de l'équation d'OrrSommerfeld pour analyser les longueurs d'onde spatiales et temporelles générées par la couche de mélange qui siègent devant les cavités.

Tout d'abord, nous introduisons la problématique des conduites flexibles et l'analyse de quelques modèles RANS et LES. En second lieu, une étude bibliographique décrit l'état de l'art au sujet des vibrations induites par cavité. Troisièmement, nous établissons l'équation d'Orr-Somerfeld et posons le problème que nous voulons résoudre en termes de discrétisations numériques de valeurs propres généralisées basées sur des schémas aux différences finies d'ordre 4. Le choix des valeurs propres significatives parmi toutes celles fournies par la solution discrète est alors une difficulté sérieuse que nous avons résolue.
\end{abstract}

\footnotetext{
Abstract - Numerical Investigation of Hydrodynamic Instabilities in Singing Risers - Offshore transportation of gaseous fluids frequently relies on flexible pipes. Flexible pipes are obtained from the helicoidal rolling of steel tape which induces a geometrical cavity from edge to edge. Along the pipe, the gas infinitely encounters the edge to edge cavity of the same shape at regular interval. In some service conditions, acoustic vibrations of huge amplitude can arise. Indeed, in front of each cavity, there is a shear layer where Kelvin-Helmholtz instability can occur. The flow encounters a large number of cavities on its path which gives rise to a new specific problem: is there a stabilized flow?

Results of large eddy scale simulations are presented as a reference but they are very costly. This gives reason to develop a numerical solution of the Orr-Sommerfeld equation in order to analyze the temporal and spatial wave lengths generated by the shear layers in front of the cavities.

First, we introduce the problem of flexible risers and the analysis of some RANS and LES modeling. Second, a bibliographical study describes the state of the art about vibrations induced by cavity. Third, we state the Orr-Sommerfeld equation and set the problem we want to solve in terms of generalized eigen values Numerical discretizations are based on finite difference approaches of fourth orders. The choice of the relevant eigen values among all those provided by the solutions of the numerical problem is the main difficulty to be tackled.
} 


\section{INTRODUCTION}

This study is related to flexible risers used in offshore gas transport. They are used to link parts that are rigidly fixed to the seabed with other parts which are subjected to sea and wind fluctuations. Their typical length is about one kilometre.

In some service conditions in which the fluid is a dry gas, acoustic vibrations have been observed. Such a phenomenon has never been observed when the fluid is a liquid. Acoustic vibrations induce a large increase of the pressure losses which can significantly obstruct the mass flow. It has been conjectured that the periodically repeated cavities of the flexible pipe wall are to blame.

Pipe flexibility comes from the spiralling of the innermost steel tape. Flexible risers are made of several layers rolled in a complex manner. An internal steel layer holds the tube in case of internal pressure drop.

From now on, we consider only the internal layer (see Fig. I) in contact with the fluid. This layer is not waterproof. That is why it is wrapped in a plastic sheet. When pressure is set, the plastic sheet is inflated and the metallic layer is assumed to be free of its movements and constrains both sides by static pressure.

As gas flows in the pipe, it encounters a joint located from edge to edge with a mixing layer observed in front of the joint, which is called the cavity. The cavity is indefinitely repeated with approximately the same shape all along the tube wall. The clip length is 1 to $2 \mathrm{~cm}$, so that the number of clips per kilometer of riser is one hundred thousand, that is to say close to infinity.

The behaviour of the mixing layer changes from one clip to the following clip since the flow is repeatedly perturbed all along the axis by the cavities. The question we ask therefore is does a stabilized flow exist or not?

\section{DESCRIPTION OF THE CONFIGURATION}

We describe hereafter a typical configuration; flowing fluid is natural gas which can be considered as pure methane. At 170 bar, the density and cinematic viscosity of methane are $\rho=150 \mathrm{~kg} / \mathrm{m}^{3}$ and $\mu=2.10^{-5} \mathrm{~kg} / \mathrm{ms}$. In service, the velocity ranges in $[0,25] \mathrm{m} / \mathrm{s}$.

The inner diameter of the riser ranges from 3 to 20 inches. In service configuration, the Reynolds number is no less than 10 million. The sound velocity is assessed at $410 \mathrm{~m} / \mathrm{s}$, so that in the range of these parameters the Mach number remains less than 0.05 . The temperature is assumed to be $20^{\circ} \mathrm{C}$.

The characteristics of this problem are a very high Reynolds number, a very low Mach and an infinite repetition of the clips. Thus, it is a new problem with respect to the number of cavities encountered.

For a 3-inch diameter tube on which we now focus, the average length of a clip is $L=15.5 \mathrm{~mm}$ (see Fig. 1 ) and that of the cavity mouth is $L / 2$. With the depth of the cavity, the shape ratio $(L / 2) / h$ is close to one. However, considering the helix roll of the wall, the transverse extension of the cavity is close to infinity. The shape of the cavity is not rectangular but rather a horn-shape with only 2 sides.

\subsection{Remarks}

\section{Remark 1}

A plastic internal liner loses its plasticity with very low temperatures in case of cryogenic gas transport. A solution consists of removing the plastic liner and in using an internal catenary flexible pipe made of metallic bellows to withstand the very low temperature and to provide flexibility. Such corrugated pipes (see Brac [4]) are waterproof because of the metallic continuity of the catenary. Therefore, the corrugated pipe is very different from the spiralling pipe which is not waterproof and needs an outer plastic liner. Static pressure is applied only on the internal side of corrugated hoses but on both sides of spiralling risers.

Consequently, the eigen modes of the structure are different for corrugated and spiralling pipes. The results for one are not right for the other.

\section{Remark 2}

Many experiments have been made with methane flowing in a spiralling 3-inch diameter pipe, typically at 25 bar at

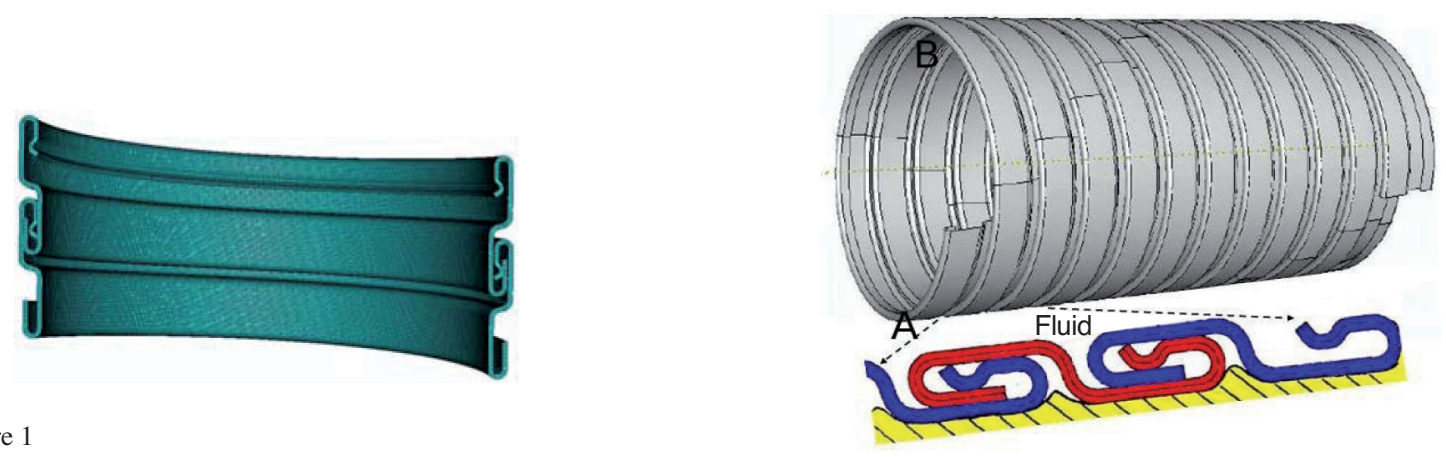

Figure 1

Set of 11 helicoidal turns showing the cavities and sketch of the clip of the innermost steel layer. 
atmospheric temperature. The test of the pipe under consideration consists in comparing the results with those of 2 other kinds of pipes of the same diameter: a smooth metallic cylindrical pipe, a PVC spiralling pipe. Some resonances correspond to the expected radial modes $(\sim 6500 \mathrm{~Hz})$ and exist with all the 3 types of tubes. Others also appear for the 3 types of pipes in the interval [2 500-4 500] Hz, in which case we conclude that they are due to a device outside the pipe. Nevertheless, we observe at $f=1300 \mathrm{~Hz}$, a typical resonance with only the spiralling pipe. This resonance has been observed during sweeps of increasing and decreasing flow velocity, with pipe in one sense and also in the opposite sense. Consequently, the riser under consideration has been observed as a singing riser.

\subsection{Outline}

The material is organized as follows:

- in Section 2, a bibliographical study describes the state of the art about vibrations induced by cavities. They could be generated by flows of various Reynolds and Mach numbers;

- in Section 3, we set the problem of flexible risers and discuss some results obtained by RANS and LES modeling. We develop a methodology to build a relevant mesh for LES modeling and to analyze the results of this numerical approach. This analysis also presents eigen modes of the structure;

- in Section 4, we focus on the instability developed at the entrance of the cavity by means of the Orr-Sommerfeld equation. We set the problem we want to solve in terms of generalized eigen value problems. Numerical features are developed through finite elements and finite difference approaches of various orders. The choice of the relevant eigen values among all the eigen value solutions of our numerical problem is a difficulty that we had to face.

Particular care is devoted to comparison with results available in the literature. Finally, an original enhancement is obtained by enlightening the results obtained by LES modeling by means of the Orr-Sommerfeld equation solutions.

\section{LITERATURE SUMMARY OF INSTABILITIES ON CAVITY}

In his classical theory for flow instability, Rayleigh [43] first developed a general linear stability theory for inviscid parallel shear flows, and showed that a necessary condition for instability is that the velocity profile has a point of inflection. Later, Tollmien [52] succeeded in showing that Rayleigh's criterion also constitutes a sufficient condition for the amplification of disturbances for velocity distributions of the symmetrical type or of the boundary-layer type.
Lin [33] pointed out the dual roles of viscosity: small viscosities have essentially a destabilizing effect while an increase of Re improves stability. In high viscosity (low Re) flow, viscosity dissipates energy at a level which stabilizes.

To sum up, it is now well-known that the presence of an inflection point on the velocity profile of an inviscid flow is a sufficient and necessary condition for hydrodynamic instability, but the role played by viscosity is complex and remains partially unknown. The acoustic wave generated by the instability is currently called Tollmien-Schlichting wave.

When a flow passes in front of a cavity, a shear layer appears and an inflexion point has to be present in the velocity profile because there is a main eddy with reverse velocity inside the cavity. This shear layer is the virtual interface of separation between the main flow and the flow locked in the cavity. Michalke [35-37] considered the thickness of the shear layer by means of a th-velocity profile and later Tam and Block [50] and Larcheveque et al. [24-25] took into account a momentum thickness.

Going deeper into fluid flowing in front of a cavity, we distinguish three kinds of phenomena both linked with the pressure:

- convection phenomena such as eddies moving at the flow velocity; these make the vorticity field crowded; they represent a field force moving on the flow and able to impact on the downstream part of the wall of the cavity. Besides, the solid structure which encloses the flow, has its own eigen frequencies. Consequently, a tonal phenomenon generated inside a flow, can stimulate an eigen vibration of the structure and awaken it. Generally speaking, it is not easy to know if the causality is included in a convection phenomenon or in an acoustic phenomenon as soon as the structure itself radiates acoustic waves in the fluid acting as added mass. This phenomenon is classified as fluid-structure interaction;

- acoustic waves which are pressure waves (compression and dilatation) travelling at the sound velocity (remember that shear waves cannot exist in a fluid) $[18,20]$. The acoustic phenomenon is then due to the geometry of the cavity where an acoustic resonance takes place. A comb of modes can be determined. Tam and Block [50] indicates $M=0.2$ as an upper limit for such phenomenon. Larcheveque et al. [24] refer to this class of phenomena as fluid-resonant. (see also Ricot's thesis [44]);

- acoustic radiation can also be linked to the shear inside vortices; the frequencies they can radiate during convection depend on the scale of the vortices. They can impact the wall, somewhere on the downstream part of the cavity and a much stronger and tonal acoustic emission can then occur. Rossiter [47] considers an acoustic feedback which perturbs the upstream entrance of the cavity. This perturbation plays the role of a conductor to synchronize the traffic on the shear layer and clamps an auto-oscillation 
at a tonal frequency depending on the main flow. Larcheveque et al. [24-25] carefully develop this mechanism which takes place when $M>0.2$ and classify this kind of phenomena as fluid-dynamic.

It can be noted that instabilities always enrich the vorticity field but the existence of an acoustic radiation depends on the energy of the vortices which is difficult to forecast. This contribution is opposite to the downstairs Kolmogorov's scales which say that "for a given scale, vortices only provide energy to vortices of immediately smaller scales". Indeed, in case of instability, vortices enlarge their own scale and reinforce their energy content along the shear layer.

In terms of modeling, convection phenomena can be pointed out by means of an incompressible formulation if Mach $<0.15$ (flow not altered by acoustic radiation) even if acoustic radiation phenomena themselves can only be modelled by means of a compressible formulation (flow altered by acoustic radiation).

Elegant approaches using the Lighthill [29-31] equation have been studied. The main idea is to locate the acoustic point sources in an incompressible model and assess their energy level by means of the Lighthill tensor and in a next step, compute Green's functions of their far field radiation. Ffowcs Williams and Hawkings [14], Powell [41] and Howe [19] adopt similar approaches using the Lighthill tensor.

\subsection{Flow Dynamic Considerations}

Rossiter [47] provides the Strouhal number of the auto oscillation of the shear layer (see Fig. 2).

The oscillation includes respectively a convection go and an acoustic back such that period $T$ and Strouhal number $S t$ are formulated as:

$$
T=\frac{1}{f}=\frac{L}{U_{c}}+\frac{L}{c-U_{\infty}} \quad S t=\frac{f L}{U_{\infty}}=\frac{n-\alpha}{M+\kappa}
$$

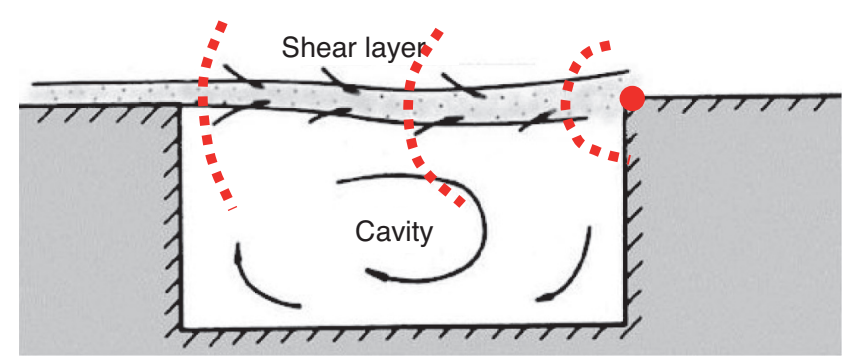

Figure 2

Mixing layer in front of a cavity according to [47]. Acoustic back from down stream corner is drawn in red wave fronts.

\begin{tabular}{c|l|c|c}
\hline$L$ & Cavity length & $c$ & Sound velocity \\
\hline$U_{\infty}$ & Velocity far from the cavity & $M=\frac{U_{\infty}}{c}$ & Mach of the flow \\
\hline$U_{c}$ & Convection velocity & $\kappa=\frac{U_{c}}{U_{\infty}}$ & $\quad(\kappa=0.57)$ \\
\hline$n$ & \multicolumn{2}{|l|}{ Number of eddies simultaneously on the shear layer } \\
\hline$\alpha$ & \multicolumn{2}{|l}{ Time delay between eddy impact and beginning of acoustic radiation } \\
\hline
\end{tabular}

Rossiter takes into account flows with Mach numbers greater than 0.4 (if $M<0.4$, the acoustic back travel is close to negligible). It can be noticed that only the length of the cavity influences the period. Thus, the tonal frequency of the unstable flow in front of the cavity can be expressed in terms of an empirical correction with two empirical coefficients $\alpha$ and $\kappa$.

Bilanin and Covert [7] propose a calculation of the coefficients $\alpha$ and $\kappa$. The mixing layer is considered as a vorticity line excited at the upstream corner. Therefore, its fluctuations mean that the cavity has been filled in and emptied half a period later. This in and out phenomena is considered to be the source of the acoustic radiation. Bilanin and Covert's formula is globally the same as Rossiter's formula but free of empirical parameters; in particular, it is more accurate at lower Mach.

Tam and Block [50] have enhanced the Bilanin and Covert's formula accounting for the cavity depth $h$ as well as the thickness $\theta$ of the momentum layer which is not yet considered as just a vorticity sheet.

The mixing layer is subject to oscillations and impacts the downstream corner at full strength if in a low position (see Fig. 3); in such a case, an acoustic radiation can occur. When the mixing layer is in a high position (see Fig. 3) [17], it has a weak impact on the corner and no acoustic radiation is emitted.
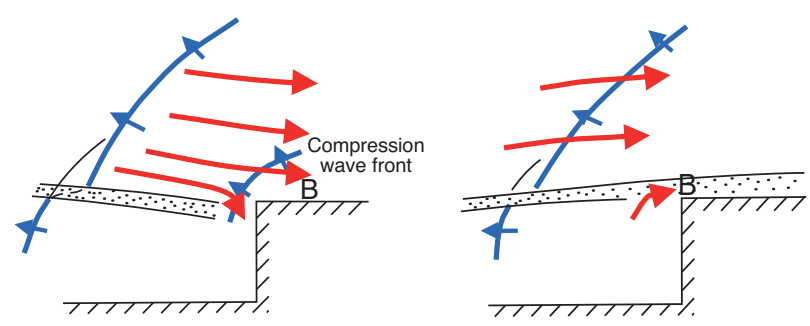

Figure 3

From [50], on the left, the mixing layer dives into the cavity and hits the wall at full strength. On the right, above the down stream, the vortices do not impact on the wall. 
They state that the ratios $L / h$ and $L / \theta$ play an important rule for the acoustic radiation. When $M \geq 0.2$, the Tam and Block formulation is relevant. For smaller $M$, the relationship to assess the Strouhal number seems to be very different from those for greater $M$. Their results are no longer correlated with the experimental results; the underlying phenomena are not the same if $M$ is smaller or greater than 0.2 . When $M \leq 0.1$, the acoustic resonance inside the cavity volume is a preferential mode. This filling/ejection mechanism is also described by Gharib and Roshko [15] as a wake mode.

Moreover, some authors such as Karamcheti [22] stress the major influence of the state of the incoming boundary layer on the level of the mixing layer fluctuation. Larcheveque et al. [24] more recently acknowledge this important remark and point out a higher level of momentum growth rate at the entrance of the cavity.

Plumbee et al. [40] suggests that the tonal noise comes from the turbulence and the structure initiates the resonance by taking its energy from a small part of the large spectrum of the turbulence. It is now widely admitted that this is impossible.

In conclusion, in case of flow on cavity, three kinds of phenomena have been classified: flow-structure, flow-resonant and flow-dynamic. Literature indicates that flow-resonant coupling is not very probable if $M<0.15$. However, the mixing layer can provide a rich vorticity field which can generate flow-structure or flow-resonant coupling in case of low Mach.

Some interesting summaries of flow on cavity are to be found in Larcheveque et al. [24], Peters [39], Powell [41] and Leonard [27] for vortex methods.

\subsection{Analysis Tools}

A set of widely used tools for instability analysis, includes:

- momentum and vorticity thickness of the shear layer of the average flow, Sarohia [48];

- pressure spectra of points chosen inside the shear layer of the average flow;

- correlations and spectra of the shear layer extracted from LES, Larcheveque et al. [24];

- Schlieren visualizations of convective and acoustic phenomena;

- coherent structures displayed by means of the $Q$ and $\lambda_{2}$ criteria, Brac et al. [3], Jeong and Hussain [21];

- analysis of the velocity and Reynolds stress profiles of RANS and LES modeling, Larcheveque et al. [24];

- phase analysis and measurement (correlation between different space locations), Sarohia [48] and Larcheveque et al. [24].

By means of the momentum thickness, a growth of the mixing layer can be pointed out; the frequencies and their harmonics can be found in the pressure spectra at a set of locations in the shear layer.
Schlieren is very expressive in terms of wave front and vortex object.

Horizontal and vertical velocity component profiles point out the inflow/outflow process in the cavity mentioned previously.

$Q$ and $\lambda_{2}$ criteria provide the contouring of the coherent structures and allow their trajectories and their impact on the downstream corner of the cavity to be followed.

The contribution of the LES modeling to enlighten the instability phenomena due to the cavity is definitively stated in [21].

Note also in [24] the calculation of the spectra by means of Burg's parametric estimator based on a maximum entropy method. This method aims to decrease the small values by smoothing the noise, increasing the peaks and enhancing their resolution in the case of short-time samples.

\subsection{Contribution of the Orr-Sommerfeld Equation}

The instability in the shear layer in front of a cavity is linear at the beginning (see [51, 52], Sect. 1 of Drazin and Reid [13] and Lesieur [28]) and then becomes non-linear when the mixing sheet rolls on itself. The Orr-Sommerfeld equation models such instability and is obtained by introducing a perturbed velocity profile in the Navier-Stokes equation and then, by simplifying the terms at the first order of the perturbation (details described in Annex):

$\left(U-\frac{\beta}{\alpha}\right)\left(\varphi^{\prime \prime}-\alpha^{2} \varphi\right)-U ” \varphi=-\frac{i \mu}{\alpha \rho}\left(\varphi^{(4)}-2 \alpha^{2} \varphi^{\prime \prime}+\alpha^{4} \varphi\right)$

In the case of inviscid gas, the equation is also known as Rayleigh's equation, a simpler one since the second member becomes zero:

$$
\left(U-\frac{\beta}{\alpha}\right)\left(\varphi "-\alpha^{2} \varphi\right)-U " \varphi=0
$$

\subsubsection{Michalke's Previous Works}

Michalke solves Rayleigh's equation and his results are written in his three papers [35-37].

He considers an inviscid fluid; the th-type velocity profile is unlimited and provides an inflexion point in the perpendicular direction to the main stream:

$$
\left\{\begin{array}{c}
U(y)=\frac{1}{2}(1+\operatorname{th}(y)) \\
y_{\min }=-\infty \\
y_{\max }=+\infty
\end{array}\right.
$$

By means of a change of variable:

$$
\varphi(y)=e^{\int_{0}^{y} \Phi(z) d z}
$$


Rayleigh's equation depending on two complex numbers $\alpha$ and $\beta$ and a complex function $\Phi$ becomes (see [43]):

$$
\Phi^{\prime}=\alpha^{2}-\Phi^{2}+\frac{U^{\prime \prime}}{U-\frac{\beta}{\alpha}}
$$

with $\Phi(+\infty)=-\alpha$ and $\Phi(-\infty)=+\alpha$.

\subsubsection{The case of Temporal Perturbation}

Michalke changes variable and uses the result of Tatsumi and Gotoh [51] stating that $\frac{\beta_{r}}{\alpha}=\frac{U(+\infty)+U(-\infty)}{2}$. It leads to:

$$
\frac{d \Phi}{d z}=\frac{\alpha^{2}-\Phi^{2}}{1-z^{2}}-\frac{2 z^{\prime}}{z-2 i \frac{\beta_{i}}{\alpha}} \text { with } \Phi(-1)=+\alpha \text { and } \Phi(1)=-\alpha \text {. }
$$

Separating real and imaginary components, the system of equations becomes a system of coupled Ricatti equations:

$$
\begin{aligned}
& \frac{d \Phi_{r}}{d z}=\frac{\alpha^{2}-\Phi_{r}^{2}+\Phi_{i}^{2}}{1-z^{2}}-\frac{2 z^{2}}{z^{2}-\left(2 \frac{\beta_{i}}{\alpha}\right)^{2}} \\
& \Phi_{r}(1)=-\alpha \text { and } \Phi_{r}(-1)=+\alpha \\
& \frac{d \Phi_{i}}{d z}=\frac{-2 \Phi_{r} \Phi_{i}}{1-z^{2}}-\frac{4 \frac{\beta_{i}}{\alpha} z}{z^{2}+\left(2 \frac{\beta_{i}}{\alpha}\right)^{2}} \\
& \Phi_{i}(-1)=+\alpha \text { and } \Phi_{i}(-1)=0
\end{aligned}
$$

Michalke gives the eigen shapes of $\Phi_{r}(z)$ and $\Phi_{i}(z)$ and the $c_{i}=\frac{\beta_{i}}{\alpha}$ values.

The max value of $\alpha c_{i}(\alpha)$ corresponds to the temporal frequency the most probably able to live in the system and Michalke provides $\left[\alpha c_{i}(\alpha)\right]_{\max }=0.2067$.

\subsection{Conclusions}

Many papers have considered flow on cavity for almost a century. The cavity has different shape ratios but there is nothing about a cavity infinitely repeated in the stream direction. The flow dynamic coupling is the most studied for flow with $M>0.2$ and high Reynolds number; many tools are described and their accuracy and efficiency are pointed out to analyze the compressible Navier-Stokes solutions of both RANS and LES modeling.

The literature also suggests that our configuration is beyond the scope of fluid-dynamic coupling since $M \sim 0.05$. The high value of the Reynolds number has no influence on the kind of coupling. A fluid-structure coupling is considered possible and the eventuality of a fluid-resonant coupling is still open; our configuration which is based on the repetition of the cavity, has so far never been investigated in the literature.

\section{NUMERICAL MODELING}

LES simulations have been carried out in several works to analyze the pressure, velocity and vorticity fields around the cavity. Care has to be taken for the meshing (see [3]); first of all, we therefore explain our methodology for LES meshing. Then, we investigate the possibility of fluid-resonant coupling due to the geometry of the cavity. Thirdly, RANS and LES modeling are analyzed with particular focus on the vorticity field and on the shear layer momentum thickness. Finally, the structural point of view is detailed.

\subsection{Discretization of the Model}

A coarse mesh is made and then RANS modeling is carried out in order to map $k$ and $\varepsilon$ values. Then, the maps of turbulence scales are managed; we deduce an interval of scale variations for integral scale $L=\frac{k^{3 / 2}}{\varepsilon}$, Taylor scale $\lambda=L \operatorname{Re}_{L}^{-1 / 2}$ and Kolmogorov scale $\eta=\left(\frac{\mu^{3}}{\rho^{3} \varepsilon}\right)^{1 / 4}$ with $\operatorname{Re}=\frac{L \rho v_{r}}{\mu}$ ( $v_{r}$ is the rotation speed of the vortex).

On pipe axis, $k=1.6 \mathrm{~m}^{2} / \mathrm{s}^{2}$ and $\varepsilon=0 \mathrm{~m}^{2} / \mathrm{s}^{3}$; the largest values are located in the cavity vicinity $k=5 \mathrm{~m}^{2} / \mathrm{s}^{2}$ and $\varepsilon=20000 \mathrm{~m}^{2} / \mathrm{s}^{3}$. The variation intervals of the scales are $6 \cdot 10^{-4}<L<3 \cdot 10^{-2}, 5 \cdot 10^{-5}<\lambda<6 \cdot 10^{-4}, 3 \cdot 10^{-6}<\eta<10^{-5}$ in meters.

The mesh is built so that the cell size is everywhere smaller then $L / 2$ (at least, 2 cells per wave length locally equal to $L$ ) and in the vicinity of the cavity, we endeavour to make cell sizes of Taylor scale size. We remark that the strongest constraint comes from the boundary layer since $1 y^{+}=2 \mu \mathrm{m}$, that is to say, less that the Kolmogorov scale (see [26, 45, 49]). Consequently, we get about one hundred cells along the mixing layer. RANS modeling is rerun using this new mesh until it satisfies all these specifications. Then LES modeling can be undertaken with the resulting mesh (see Fig. 4).

In order to stabilize turbulence of the flow, the velocity field of the output section is sequentially set at the input section, so that input and output velocity fields are identical when converging.

Since a geometry pattern is identically repeated in the $z$ direction, the pressure is periodic in the sense that the pressure drop inside a pattern of length $L$ including an integer number of cavities is such that:

$$
\Delta p=p(z)-p(z+L)=\ldots=p(z+(n-1) L)-p(z+n L)=\ldots(6)
$$




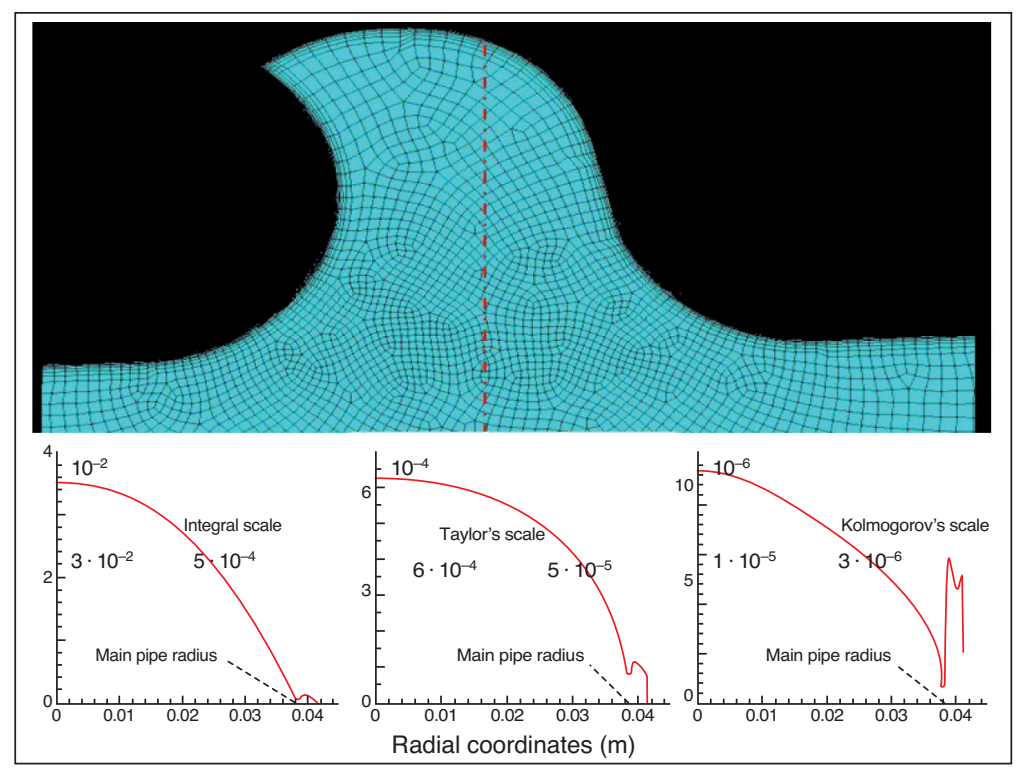

Figure 4

Integral, Taylor and Kolmogorov scales through a radius ending at the bottom of the cavity (red dotted line). The variation interval is indicated for each scale.

Density is constant and $\Delta p$ is assumed to be a constant value, in stabilized flow, that is to say a large number of cavities have been encountered. Thus, the local pressure gradient is:

$$
\nabla p(z)=\beta \frac{\vec{L}}{|L|}+\nabla \tilde{p}(z)
$$

and it can be broken down into two parts:

- $\beta \frac{\vec{L}}{|L|}$ is the linearly-varying component of the pressure,

- the gradient of a periodic component $\nabla \tilde{p}(z)$ where $\tilde{p}(z)$ is the periodic pressure field.

The periodic pressure is the pressure left over after subtracting out the linearly-varying pressure. The linearlyvarying component of the pressure results in a force acting on the fluid in the momentum equations. Because the value of $\beta$ is not known a priori, we iterate until the specified mass flow rate (imposed through the averaged velocity) is achieved in the computational model.

The periodic pressure field of the input section is set at the output section in order to also make the residual pressure field periodic. The model is therefore assumed to mimic a domain of infinite length. Such periodicity is only possible if the model includes an integer number of helix turns in order to have exactly the same meshing on the input and the output sections.

In conclusion, the mesh for the LES model is sensitive and we propose a method to build such a mesh. Starting from a coarse RANS modeling mesh, we evaluate the turbulence wave length and fit each cell to be locally twice smaller than the integral scale. Iteratively, we converge to an LES appropriate meshing. Moreover, with such periodic boundary conditions, the problem is well-posed.

\subsection{Helmholtz-Type Tube Resonance}

We consider 2 types of acoustic resonance induced by the geometry of the tube: longitudinal and radial modes. The question under interest is (see [42]): does helix cavity alter theses modes or not? Has it an own eigen mode? (see [2] for acoustic in general [5, 6, 8-12]). Moreover, we focus on the interval $I_{1}=[100,3000] \mathrm{Hz}$ which included the frequency $1300 \mathrm{~Hz}$ where a resonance has been experimentally observed (pipe of 3' diameter).

Longitudinal modes: one turn of helix has a given length $L$ and the numerical model consists of $n$ turns. Frequencies depending on the average velocity $\bar{u}$ and equal to $\mathrm{f}=\bar{u} /(n L)$ would be present in the solution. They depend on the length of the model and have been identified in the spectrum. For one turn, we have $(15 \mathrm{~m} / \mathrm{s}, 1050 \mathrm{~Hz}),(20 \mathrm{~m} / \mathrm{s}, 1400 \mathrm{~Hz}),(25 \mathrm{~m} / \mathrm{s}$, $1745 \mathrm{~Hz}$ ). When the model includes $n$ turns, these frequencies are divided by $n$. Seven turns are needed to have $L>D$ and the basic frequency is then $155 \mathrm{~Hz}$ at $15 \mathrm{~m} / \mathrm{s}$. The basic frequency tends to zero when the tube length becomes large. These longitudinal modes and their harmonics are present in the $n$ turn mathematical model but they cannot be observed in experimentation with tube of different and greater lengths. 

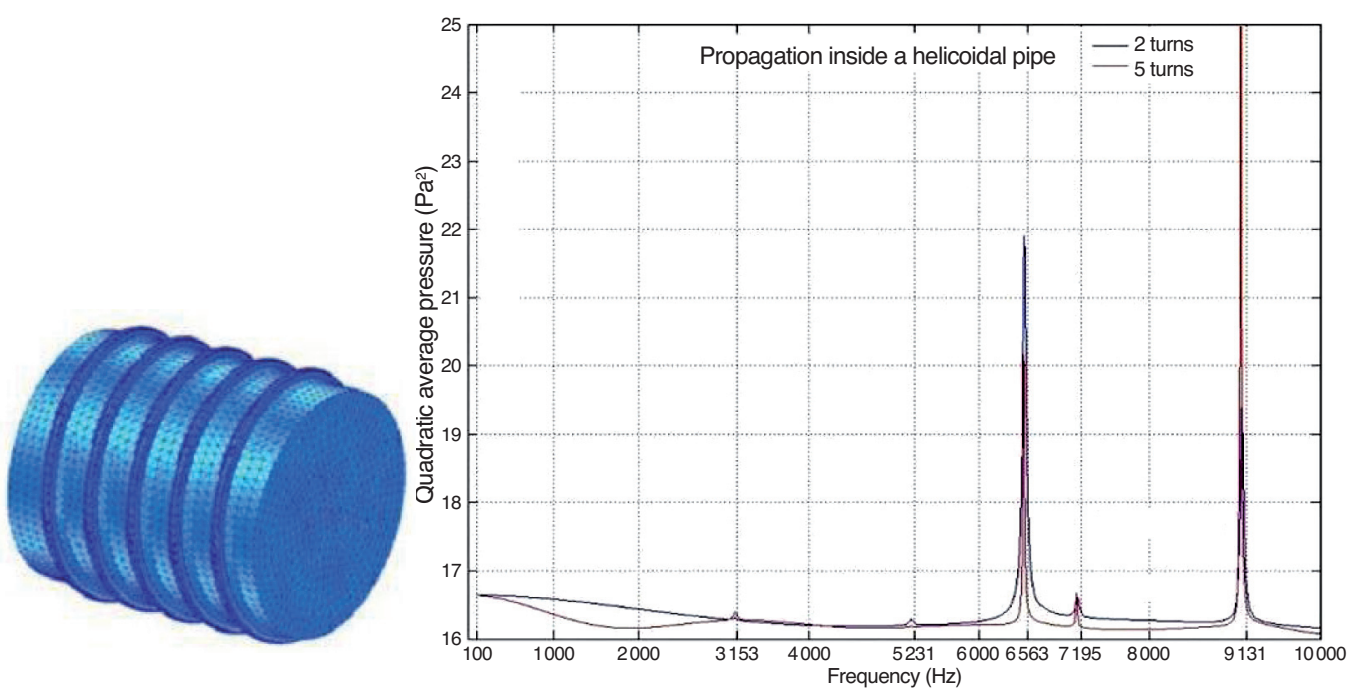

Figure 5

5 turns model on the left; quadratic pressure spectrum in a tube of 3' diameter with 2 and 5 clips respectively. Note the radial mode at $3153 \mathrm{~Hz}$ and the strong first harmonic at $6563 \mathrm{~Hz}$.

The longitudinal acoustic mode is $28621 \mathrm{~Hz}$ for one turn. Therefore, the longitudinal acoustic modes are out of scope.

Radial modes: the radial modes are given by:

$$
f_{n, m}^{r}=\frac{\chi_{n m} c}{2 \pi r}
$$

where $\chi_{n m}$ represents the $m$ th zero of the derivative of the Bessel function of order $n$; namely, $\chi_{11}=1.84$.

For a three-inch diameter riser, the first radial acoustic mode $f_{11}^{r}=3150 \mathrm{~Hz}$ is greater than $3000 \mathrm{~Hz}$ and does not depend on the number of turns (see Fig. 5). Accurate studies of the Helmholtz-type resonance of the tube with and without the cavities have been carried out using the Far Field Technologies software. It states that the cylindrical radial modes are not meaningfully altered by the presence of the helicoidal cavities.

Consequently, on the interval $I_{1}$ under consideration, the radial mode resonance is out of scope. The fluid-resonant mode is excluded for interpreting phenomena whose frequencies belong to $I_{1}$.

\subsection{RANS and LES Modeling}

RANS and LES modeling are carried out using Fluent's solver. The sub-grid scale model is that of Smagorinsky-Lilly $\left(C_{s}=0.1\right)$; a "bounded central differencing scheme" is used for the momentum balance equation and a wall law avoids a very fine mesh at the wall. Thus, boundary law is activated as long as the thickness of the first cell at the wall ranges from 12 to $\sim 300 y^{+}$about (see [23] for more details about boundary layer). Time step is $d t=5 \mu \mathrm{s}$. Before having the stabilized flow regime, we carry out as many steps as needed to pass through at least 20 times the model length. This procedure is very costly and with a model of 11 turns (35 million of hexahedral cells), 2 weeks on 64 processors are necessary to get the stabilized flow.

In Figure 6, LES and RANS velocity profiles are displayed along a diametric line $\mathrm{AB}$ passing through a cavity. Numerous fluctuations can be observed from one instant to another. LES curves are snapshots frozen at successive times. The corresponding RANS profile shows a coarser evolution of the velocity; there is a close-up of the profile inside the cavity on the right. Lots of inflexion points of the instantaneous curves are averaged in the RANS profile and most of the nervous inflexions disappear. One of them is maintained (see Fig. 6c) at the entrance of the cavity. The reverse velocity is clearly visible inside the cavity; it points out a loop of fluid designed by the cavity geometry.

\subsection{Axial and Transverse Evolutions of the Vorticity Field}

Figure 7 shows four snapshots of the oscillations of the mixing layer in front of a cavity like the flapping of a sail in the wind (4 snapshots of the radial derivative of the axial component of the velocity). Figure 7 focuses on the upstream corner where eddies rise. 


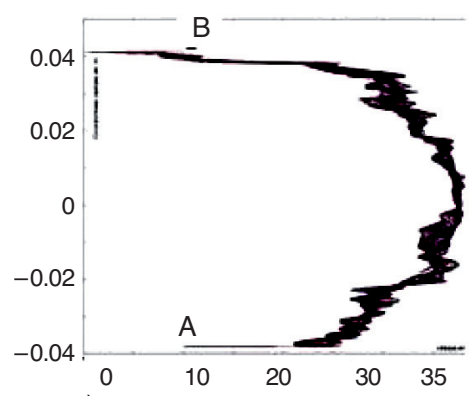

a)

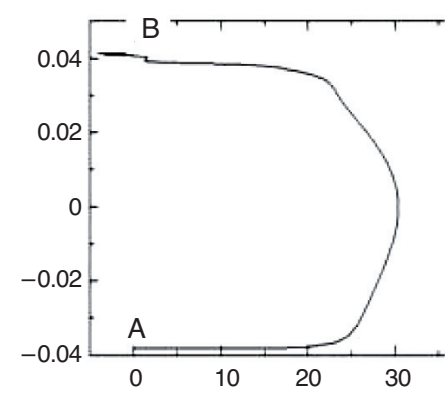

b)

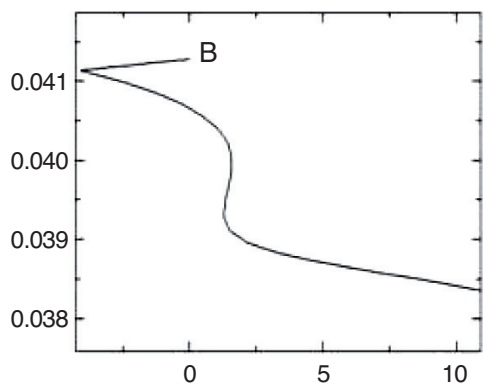

C)

Figure 6

Axial velocity component in m/s along the diameter A-B in m (for A, B locations, see Fig. 1). a) Many superimposed instantaneous velocity profiles of LES modeling; b) same profile of RANS modeling; c) close-up in the neighborhood of B.

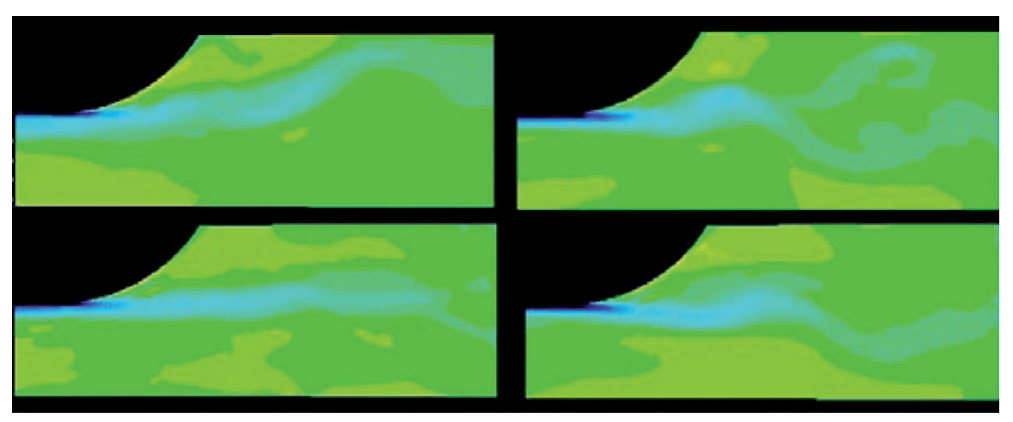

Figure 7

4 snapshots of the radial derivative of the axial velocity component in the vicinity of an upstream corner of cavity.

Figure 8 displays the eddies according to the $\lambda_{2}$ criterion as described in [3].

We observe the detachment of the eddies on the left, their growth along the mixing layer and their impact against the wall on the right. We also observe their lateral spreading which is not disturbed since the transverse size of the cavity is infinite.

The Helmholtz or vorticity equation is easily deduced from the momentum balance NS equation; it is written as:

$$
\frac{\partial \omega}{\partial t}-u . \nabla \omega=\omega . \nabla u+\frac{\mu}{\rho} \Delta u
$$

Both terms on the left side of the equation are the convective derivative of the vorticity vector. The term $\frac{\mu}{\rho} \Delta u$ corresponds to a viscous dissipation while $\omega . \nabla u$ indicates a spread of the vorticity lines in the transverse direction; it is experimentally observed and Figure 9 illustrates this evolution. That is why $2 \mathrm{D}$ turbulence modeling is not relevant.

This snapshot shows the continuous mixing layer beneath successive clips; a helicoidal component of the eddy trajectory can be observed. The Kelvin Helmholtz instability generates unsteady phenomena with poor continuity. What happens in the cavity is very different from one cavity to another.

The Lighthill tensor mapping offers the location of the acoustic sources but also the positions of the centers of high and low pressure, depression and anticyclone. If $T_{i j}$ is the Lighthill tensor, then the Lighthill equation is (see [29-31]):

$$
\frac{\partial^{2} \rho}{\partial t^{2}}-c_{0}^{2} \Delta \rho=\frac{\partial^{2} T_{i j}}{\partial x_{i} \partial x_{j}}
$$

The following map shows the contouring of $T_{i j}$ at a given instant in two radial sections. A traffic of two or three vortices is displayed and illustrates the lateral variability of the vorticity field. The blue-green area can emit acoustic waves but the emission is low level and is not the basis of the phenomena we observe in Figure 10.

\subsection{Evolution of the Thickness of Shear Layer Momentum Quantity}

The thickness of momentum is defined by the means of the following integral along the radial coordinate $r$ from the axis 

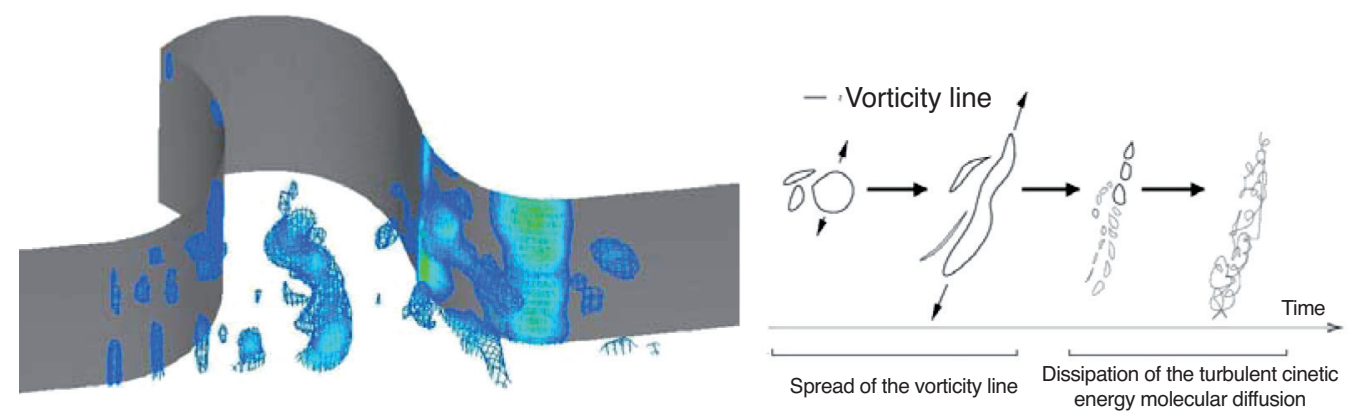

Figure 8

Snapshot of the vorticity field displayed by means of the $\lambda_{2}$ algorithm; on the right, sketch of vortices evolution in the transverse direction to the flow $[3,34]$.

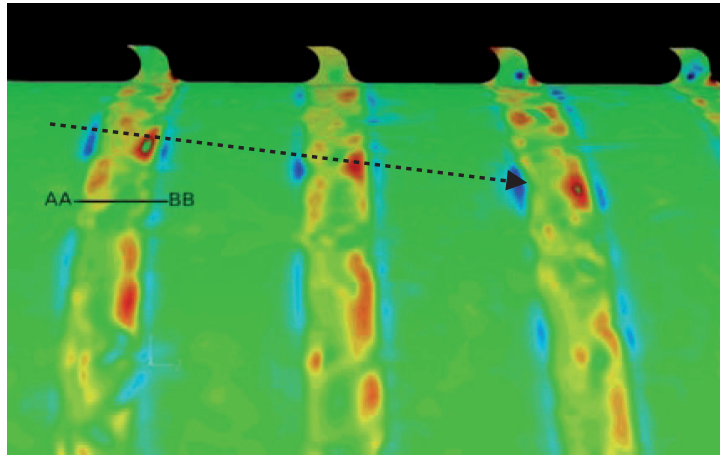

Figure 9

Circumferential sections of static pressure displaying the mixing layer and the lateral spreading of the vortices.

of the tube to the bottom of the cavity where the axial ordinate $z$ is fixed:

$$
\theta(z)=\int_{a x i s}^{\text {wall }} \frac{u(z, r)}{\bar{U}}\left(1-\frac{u(z, r)}{\bar{U}}\right) d r
$$

$\theta_{0}$ is the value of the thickness when $z<<z_{A}$ and $z>z_{D}$.

Such calculation can be performed on maps of velocity issues of RANS (as indicated in the literature summary) or LES modeling. We present momentum thickness calculation carried out on LES map of velocity; the section area has been chosen in order to be located at a place where vorticity is strong and phenomena well pronounced. In Figure 9, it is clear that at certain locations, there is no inflexion point in the velocity diametral line and consequently the momentum thickness does not vary through the cavity mouth.

Along the radial lines of Figure 11 where $U=20 \mathrm{~m} / \mathrm{s}$, we compute the values of thickness (corresponding velocity profiles shown in Fig. 12a) and have plotted the dimensionless momentum values in Figure 12b. The locations A, B, C and D are plotted on the curve. The slope of the curve seems

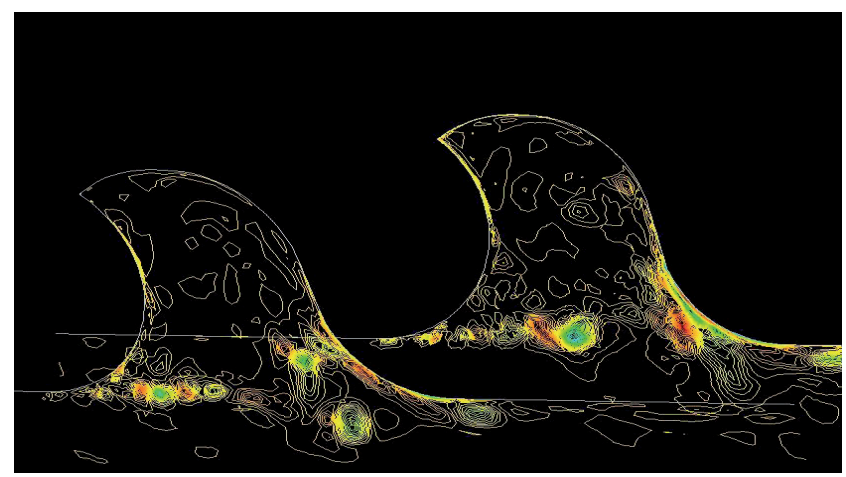

Figure 10

This snapshot represents two maps of radial section of $T_{i j}$, Lighthill tensor, extracted from LES modeling; blue and green correspond to a low pressure area and red to high pressure [19, 20, 29-31, 53].

constant between $\mathrm{B}$ and $\mathrm{C}$; it appears that the momentum thickness is $0.0175 \mathrm{~mm}$ in the flow, increases to $0.45 \mathrm{~mm}$ when passing through the cavity and goes back to $0.0175 \mathrm{~mm}$ as soon as the cavity is passed. Consequently, a stabilized flow exists when fluid-structure coupling is not taken into account.

A slope of $d \theta / d z=0.05$ can be observed. Moreover, a stronger slope seems to be present upstream of A and also upstream of $\mathrm{C}$, indicating a larger growth rate of the momentum layer. Upstream of point A, this precursor is linked with the cavities located before the considered cavity although, upstream of $\mathrm{C}$, the larger thickness could be explained by the impact of the vortices on the wall.

In conclusion, we see a relevant level of momentum exchange between the main stream and the mixing layer since the shape ratio of the cavity is close to 1 . However, the duration of the exchange is short since the cavity is narrow compared to the diameter. 


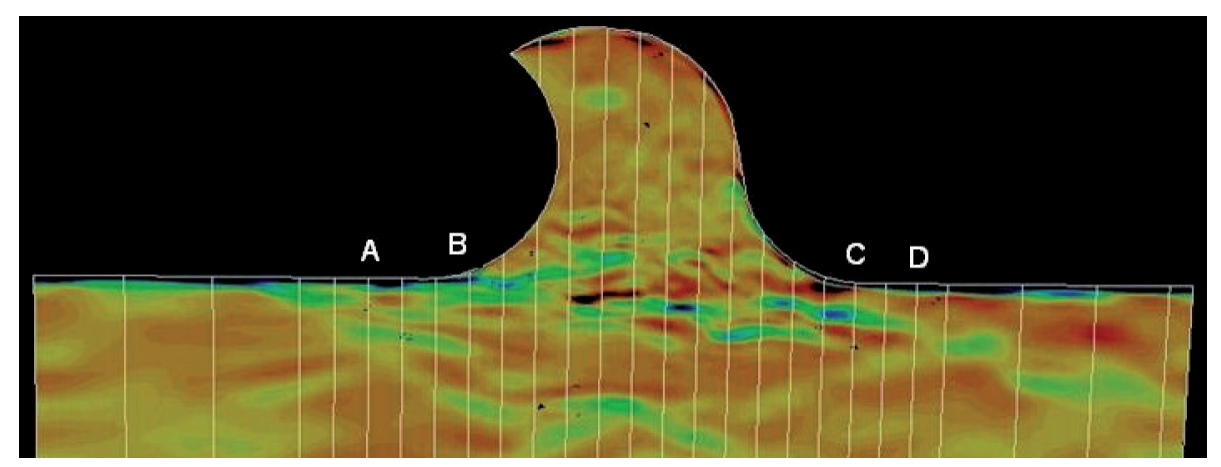

Figure 11

Set of radial lines in the vicinity of a cavity.

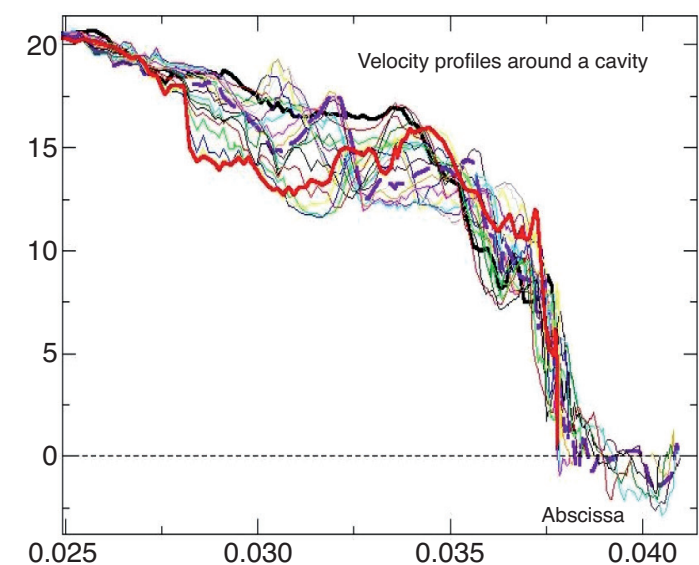

a)

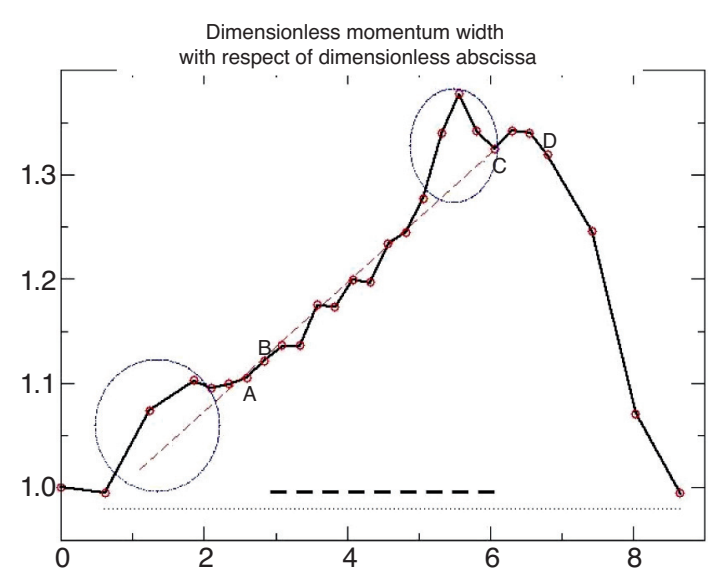

b)

\section{Figure 12}

Partial views of LES velocity profiles before, through and after a cavity; dimensionless momentum thickness $\theta / \theta_{0}$ with respect to the dimensionless axial coordinate in $z / \theta_{0}$ computed using these profiles. Points $\mathrm{A}, \mathrm{B}, \mathrm{C}$ and $\mathrm{D}$ are indicated in the previous figure.

Several trials at other locations where vorticity is strong, give similar results. For certain locations of line $\mathrm{AB}$, there is no inflexion in the velocity profiles.

\subsection{Structural Point of View}

Finally, the eddies fed by the shear layer, impact the wall at the downstream corner of each cavity. Eddies can be seen as force field able to excite the solid structure made with the steel tape as we can see in Figure 13 (deep blue spots) (see [46] for vortex-body interaction).

The applied eddies load excites the shell made by the steel tape wrapped around itself. According to the surface and the frequency of the applied forces, the modal frequencies of the structure may be requested for a structural motion, termed as mode, when the eddies hit it. An eddy has to be a negative pressure zone since the pressure gradient has to be opposed to the centrifugal force to ensure the cyclostrophic equilibrium [3]. Thus, a couple of forces is applied to the wall; it is linked with a pair of local area of positive pressure (anticyclone) and of negative pressure (vortex). Each couple is weak but the spiral wall is submitted to the integral of the forces per length unit and we count several tens of vortices per turn.

In the present study, the auto wrapped particularity of the structure urged us into paying special attention to the possibility of auto contact due to shell vibrations.

If the structure wants to develop a motion which is powered by the fluid action, nothing (and even itself) must constrain its motion in the obtained mode $[1,16]$.

To obtain the equations ruling the vibration theory of plate, one must find the harmonic motion which can solve the equations of equilibrium including the acceleration terms. 


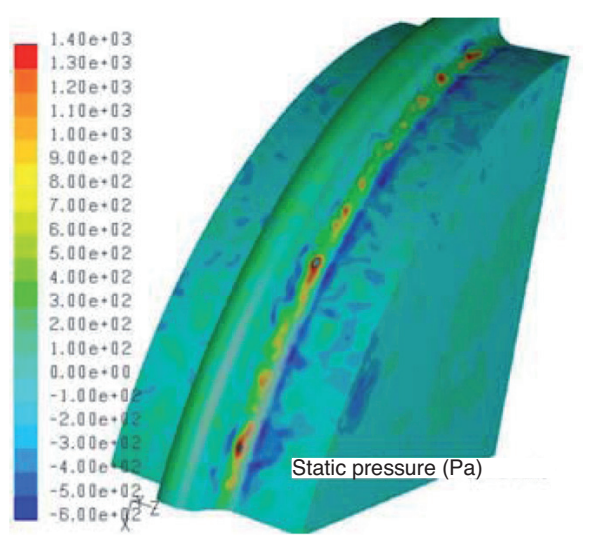

Figure 13

Pressure field at the wall on downstream side with flow at mean velocity at $15 \mathrm{~m} / \mathrm{s}$. Negative pressure in blue (vortex) and positive pressure in red (anticyclone) with respect to the reference pressure. Pressure ranges from $-10^{3}$ to $+1.5 \times 10^{3} \mathrm{~Pa}$.

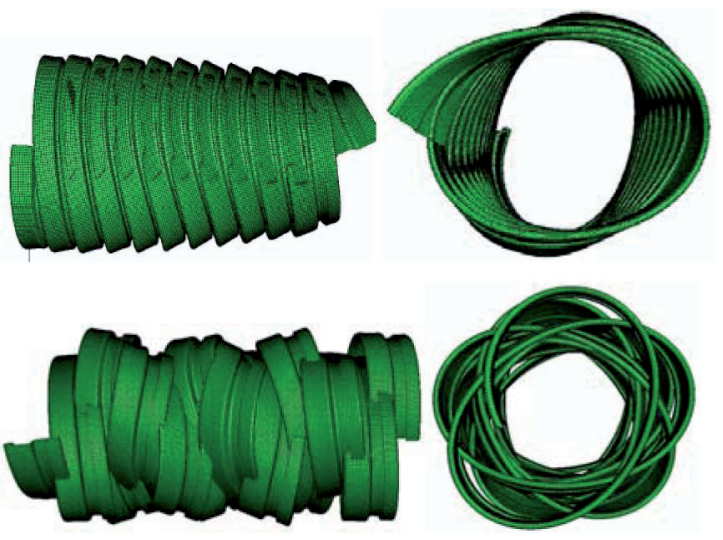

Figure 14

Views of 2 physical modes, obtained with Abaqus software. On the first row, a compatible mode; on the second row, an unphysical mode.

Notice the large magnification of the displacements.
The usual problem ends in an eigen value problem where, $M$ being the mass matrix and $K$ the rigidity matrix, the eigen value problem can be formulated as:

$$
\left(-\omega^{2} M+K\right) \varphi=0
$$

where $\omega$ is the eigen frequency linked to the eigen vector $\varphi$; the dimension of $M$ and $K$ matrices corresponds to the number of degrees of freedom of the finite element model.

The resolution made thanks to the FEM analysis software, ABAQUS, makes the modes free of constraints. But as was previously noticed, there are forbidden displacements which make penetration of material physically impossible. We filter by hand the free modes in order to avoid the penetration of the turns and keep the modes whose amplitude remains compatible with the geometry. Only a few numbers of modes result from this filtering.

One of the eigen modes of the flexible hose with large exaggeration of the amplitude is shown in Figure 14. All the realistic eigen modes assumed no overlapping of the turns between them; indeed, we know that the vibrations do not break the structure and consequently that overlap of the turns does not really exist. Such an unrealistic and rejected mode is also displayed in Figure 14.

The number of physical modes depends on the number of turns included in the model. In the real case, the pipe length can be seen as infinite. Since we did not use periodical boundary conditions with ABAQUS FEM, we ran the analysis for several turns of the helix structure. The modal analysis delivers the modal trend for 3, 5, 7 and 11 turns of the steel roll. This parameter study shows a fast converging frequency interval in terms of turn for each mode.
In the case of a 3'-diameter tube, the first interval of frequencies is $I_{1}=[1100,1450] \mathrm{Hz}$ and the second one is $I_{2}=[3050,3300] \mathrm{Hz}$. The first interval is linked to a mode which is a two-lobe pattern mode and the second is described by a three-lobe pattern mode. We focus our analysis on interval $I_{1}$ and on this specific mode which is depicted in Figure 14.

Several clip geometries have been studied. The modes depend on the thickness of the iron wall.

\subsubsection{Frequency Content of the Mixing Layer}

We intensively study a 3 turns model for three averaged velocities $15,20,25 \mathrm{~m} / \mathrm{s}$.

Figure 15 displays the static pressure field $(\bar{u}=15 \mathrm{~m} / \mathrm{s})$ along an axial line parallel to the tube axis, located $1 \mathrm{~mm}$ below the cylindrical wall and passing in front of 3 successive cavities. The slope of the event points out a convective velocity of $\bar{u}_{\text {wall }}=9.2 \mathrm{~m} / \mathrm{s}$ averaged on the line.

We deduce the frequency of the vortices on the shear layer:

$$
f_{\text {cavity } 1}=\frac{\bar{u}_{\text {wall }}}{L_{\text {cavity }}}=1285 \mathrm{~Hz}
$$

The pressure signal is fairly rough and what happens in one cavity is different to what happens in other cavities at the same time but also in the same cavity at different times. Without coupling fluid and structure, events are not well marked. However, we can observe three simultaneous vortices in the central cavity around $7 \mathrm{~ms}$.

We also consider a frequency linked to the model length:

$$
f_{\text {model } 1}=\frac{\bar{u}}{L_{\text {model }}}=698 \mathrm{~Hz}
$$




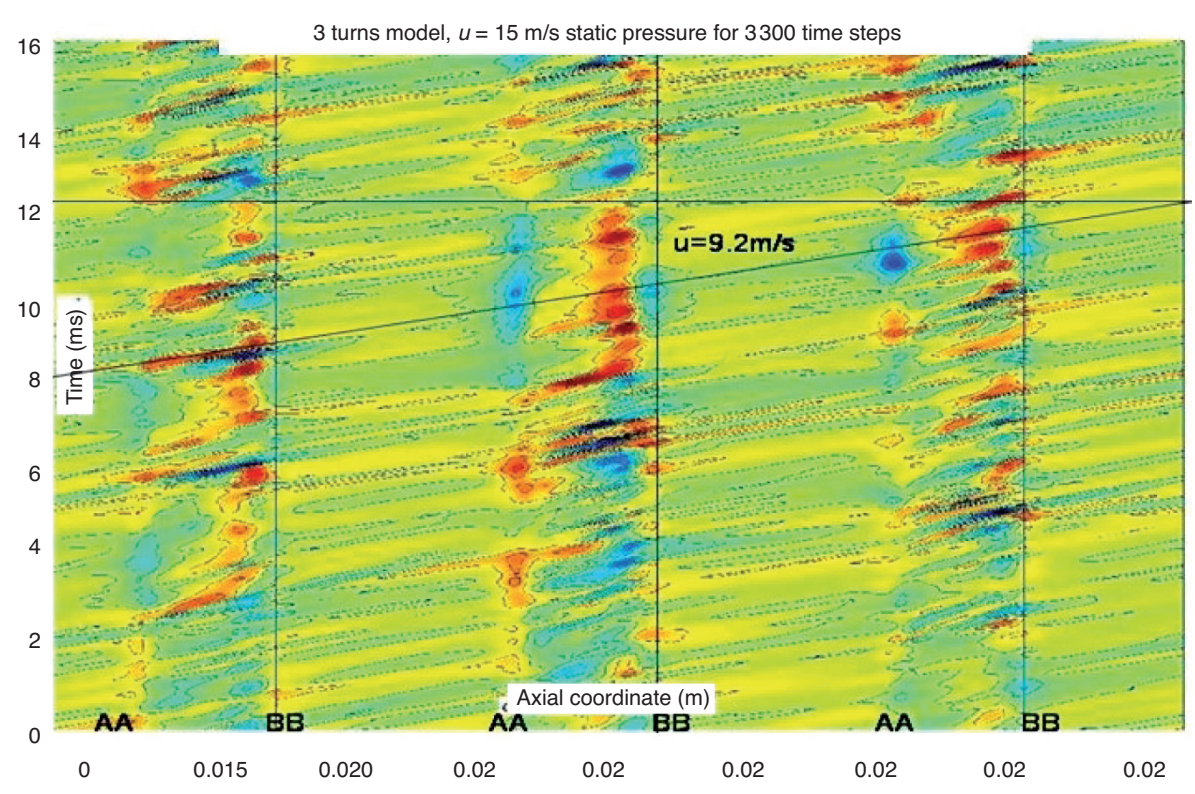

Figure 15

Static pressure records in a three turn model. AA-BB segment is at a cavity mouth. Blue corresponds to depression and red to anticyclone. Average velocity $9.2 \mathrm{~m} / \mathrm{s}$ along line AA-BB.

This frequency is spurious since it tends to zero when the model length tends to infinity. This frequency cannot be observed in service.

The results of the study with the three velocities $\bar{u}$ are gathered in Table 1 where velocities and frequencies are taken into account with respect to the model length and the cavity length. The difficulty is to assess the average velocity in the shear layer. We present results of the average velocity of an axial line. Indeed, shear layer is volumetric and deviates somewhat from just an axial line which cannot represent its whole behavior.

TABLE 1

Velocity and frequency in the whole model and on the axial line located at $1 \mathrm{~mm}$ of the wall

\begin{tabular}{c|c|c|c}
\hline $\bar{u}$ (flow) & $f_{\text {mod el } 1}$ & $\bar{u}_{\text {wall }}$ (axial line) & $f_{\text {cavity } 1}$ \\
\hline $\mathrm{m} / \mathrm{s}$ & $\mathrm{Hz}$ & $\mathrm{m} / \mathrm{s}$ & $\mathrm{Hz}$ \\
\hline 15 & 698 & 9.2 & 1285 \\
\hline 20 & 930 & 12.2 & 1703 \\
\hline 25 & 1163 & 15.5 & 2164 \\
\hline
\end{tabular}

Traffic (see Fig. 11) with one, two or three vortex exists and the scoop of this axial line is a very partial view of the volumetric vortices traffic on the shear layer (see Fig. 8). Animation shows numerous vortices on travel. Moreover, a certain amount of vorticity goes from the output of a cavity to the entrance of the next one in the stabilized flow. The momentum thickness quantifies such an amount.

For three service velocities, Figure 16 displays the frequency content (co-ordinate in $\mathrm{Hz}$ ) and their magnitude (colors) through the cavity (ordinate in $\mathrm{mm}$ ). Frequency increases with the velocity as can be observed for different groups of information that can be followed from one case to another.

The slope of the pressure events is regular from one velocity to another and indicates a constant velocity of the vortices along the mixing layer. The continuous red line of Figure 16 suggests a Strouhal $S t=2$. There is little activity of the shear layer.

The red dashed line corresponds to the frequencies due to the model length. They are spurious and tend to zero if the model length increases. That indicates $S t=0.7$ for a threeturns model.

Spectral analysis of the temporal content of an axial line extracted from LES modeling provides frequency of the cavity activity. These frequencies are not very present locally and in the numerical model, perturbed by the spurious frequencies due to the model length. However, numerous spots of impact exist all along the downstream edge of the helix cavity. Regarding the interval of eigen frequencies of the structure, $\bar{u}=15 \mathrm{~m} / \mathrm{s}$ generates frequencies in $I_{1} ; \bar{u}=20 \mathrm{~m} / \mathrm{s}$ is at the boundary of this interval and $\bar{u}=25 \mathrm{~m} / \mathrm{s}$ is outside.

The mixing layer is the area where these eddies live and their own spatial and temporal characteristics are difficult to quantify. Frequencies are able to excite the structure until 


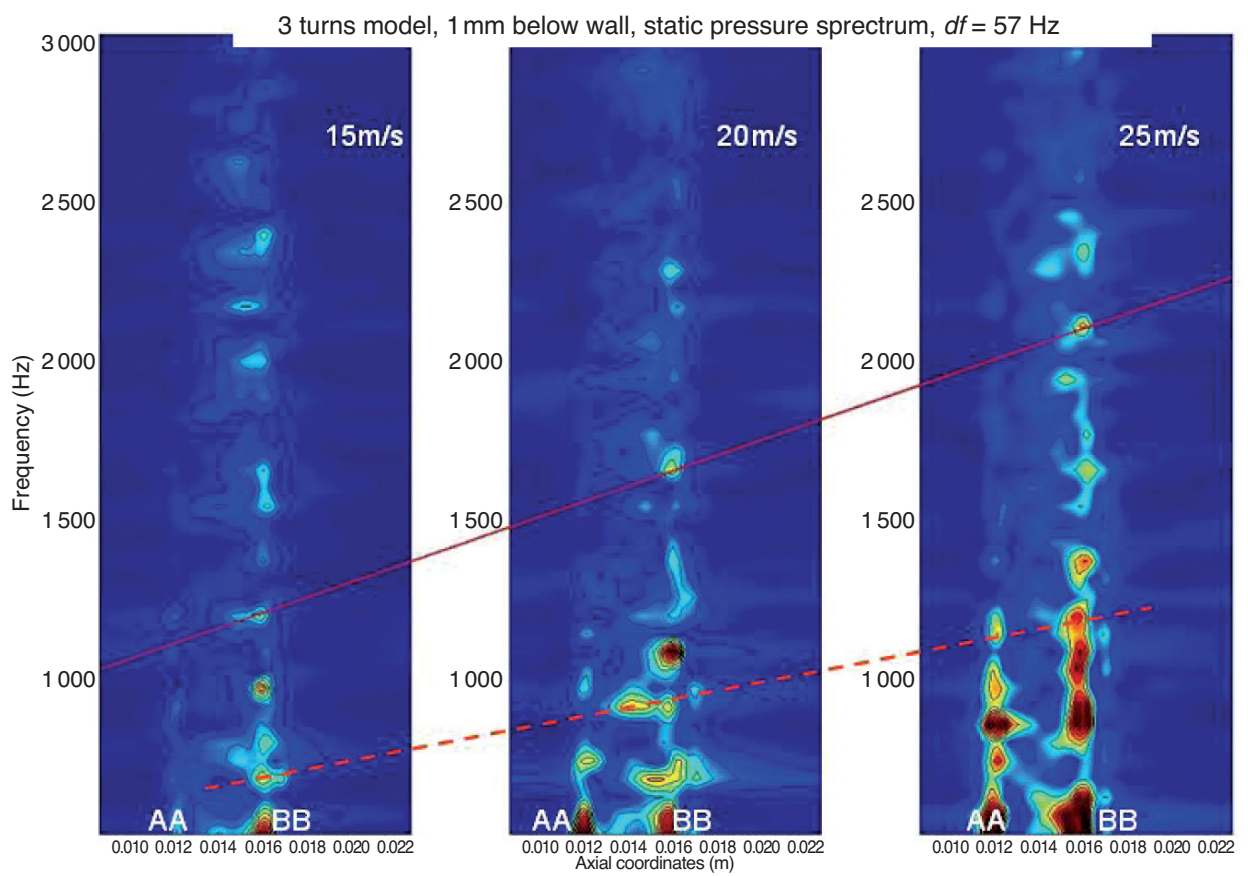

Figure 16

Spectral evolution of the static pressure at mean velocities of 15, 20 and $25 \mathrm{~m} / \mathrm{s}$ in front of 3 cavities. Red line concerns the cavity length, dotted red line the model length, frequencies depending of their location on the line in the mixing layer.

$20 \mathrm{~m} / \mathrm{s}$ and for greater flow velocity, the hydrodynamic excitation has no effect on structure.

Moreover, the cost of the incompressible LES modeling is very high and requires many processors on a cluster during a long period (typically 64 processors for a week for a threeturn model). This modeling shows the activity of the cavity shear layer. It is locally weak and frequencies able to excite the structure exist for $15 \mathrm{~m} / \mathrm{s}<u<20 \mathrm{~m} / \mathrm{s}$; for $25 \mathrm{~m} / \mathrm{s}$, hydrodynamic excitation would have no effect on structure.

\section{RESOLUTION OF THE ORR-SOMMERFELD EQUATION}

The Orr-Sommerfeld equation represents the phenomenology of a shear layer submitted to a perturbed velocity field at the first order. It seems interesting to use it in order to set an eigen value problem characterizing the stabilized flow in a flexible riser. First of all, we performed Michalke calculations using our own method. Then, beyond Michalke previous works, we set an eigen value problem and solved it.

\subsection{New Calculations: Resolution by Dichotomy}

We revisited the Michalke problem using our own numerical methodology since Michalke's numerical method is not detailed in his papers. We do not directly solve the eigen value problem; but we use an order 2 Runge Kutta method with constant step to solve Rayleigh's equation. The solution consists in a complex stream function $\Phi=\Phi_{r}+i \Phi_{i}$ and 2 complex numbers $\alpha=\alpha_{i}+i \alpha_{i}$ and $\beta=\beta_{r}+i \beta_{i}$. Temporal perturbations are found with $\alpha_{i}=0$ and the spatial perturbations correspond to $\beta_{i}=0$.

The starting point is $\Phi_{r}(z=-1)=\alpha$ and $\Phi_{i}(z=-1)=0$. We solve the problem for $\beta_{i}$ given; then, the values of $\Phi_{r}$ and $\Phi_{i}$ are found at $z=1$. We change $\beta_{i}$ value by dichotomy until the end values are correctly reached, with $\Phi_{r}(z=1)=-\alpha$ and $\Phi_{i}(z=1)=0$. Figure 17 shows that the resolution is stiff in the sense that a very small change of the $\alpha$ value changes the $\beta_{i}$ value considerably.

Remark: this problem is somewhat similar to a ballistic ray tracing problem with a given starting angle at the source; the ray ends somewhere and it can be paid with the starting angle to reach a given ending location and then solve a twopoint ray tracing problem by successive approximations.

In this way, we confirm Michalke's results obtained for inviscid fluids.

Spectral theory is a recent theory which was not wellknown when Michalke was working. We suggest writing the variational or weak formulation of Rayleigh's problem and solving it with a finite element method. 


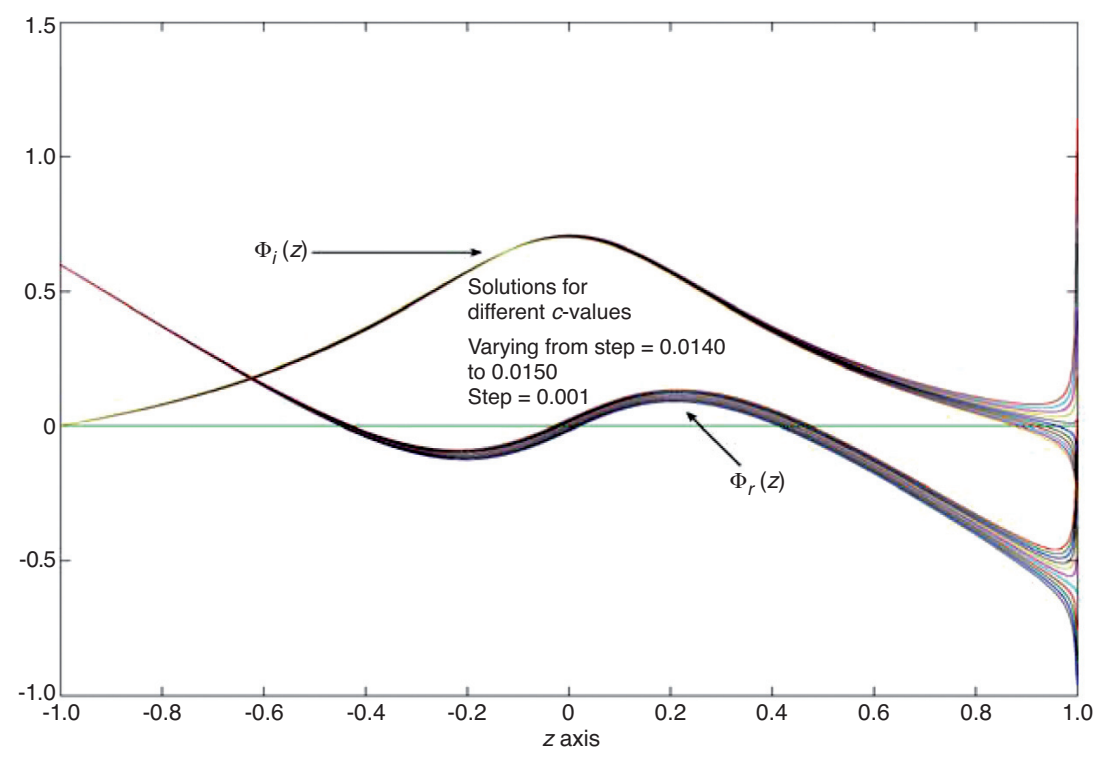

Figure 17

Real and imaginary components of $\Phi_{r}$ and $\Phi_{i}$ around a given right end value.

The boundary conditions are reached at the wall of the tube. Between two cavities, the wall is located at the range $R$ of the axis but the depth of the cavity varies $\lambda(z)$. Boundary conditions are not yet at infinity and we impose asymmetric Dirichlet's conditions at the wall for $\varphi$ at given $z$ :

$$
\left(U-\frac{\beta}{\alpha}\right)\left(\varphi "-\alpha^{2} \varphi\right)-U " \varphi=0
$$

with $\varphi(-R)=0$ and $\varphi(R+\lambda(z))=0$.

$\lambda(z)$ is the function which describes the geometry of the clip beyond the range $R$ from the axis.

Panaitescu [38] proposes a method to solve this eigen value problem by means of an second integral of Voltera's equation. This Voltera's equation represents the kernel of a linear function linked to an inferior triangular matrix. However, this elegant formulation cannot solve the eigen value problem efficiently, except to express a weak formulation of the variational problem.

\subsection{Resolution of the Orr-Sommerfeld Equation with P2 Finite Elements}

We consider a problem of temporal perturbation where $\alpha$ is real and fixed, $\beta$ is a complex number and $\varphi$ a complex function, both unknowns. The weak formulation of the Orr-Sommerfeld equation is written in the functional space $X=\varphi \in H^{2}\left(\left[-y_{\min },+y_{\max }\right], C\right)$ with $\varphi\left(y_{\min }\right)=\varphi\left(y_{\max }\right)=0$ and $\varphi^{\prime}\left(y_{\min }\right)=\varphi^{\prime}\left(y_{\max }\right)=0$ so that solving the Rayleigh problem or solving the weak formulation is equivalent. The LaxMilgram theorem enables us to be sure that there is only one solution, that is to say, the problem is well-posed. It becomes, $\forall \psi \in X$ :

$$
\begin{aligned}
& -\int_{-M}^{+M}\left(\varphi^{\prime}\left(U^{\prime} \bar{\psi}\right)+\alpha^{2} U \varphi \bar{\psi}-U^{\prime \prime} \varphi \bar{\psi}\right)+ \\
& \frac{i u}{\rho \alpha} \int_{-M}^{+M}\left(\varphi^{\prime} \bar{\psi}^{\prime \prime}+2 \alpha^{2} \varphi^{\prime} \bar{\psi}^{\prime}+\alpha^{4} \varphi \bar{\psi}\right)=\frac{\beta}{\alpha} \int_{-M}^{+M}\left(\alpha^{2} \varphi \bar{\psi}-\varphi^{\prime} \bar{\psi}^{\prime}\right)
\end{aligned}
$$

With $\mathrm{P} 2$ finite elements, the derivatives of the basis function are not continuous, so that they are not in $H^{2}$. P2 elements are not conform. It is not yet possible to find a subspace to discretize the weak formulation. It could be necessary to choose P3 elements but complexity of the calculation of the integral increases considerably. Nevertheless, we try to solve with only P2 elements and discuss later the result validity.

\subsection{Resolution of Orr-Sommerfeld Equation with Finite Differences}

We now consider the finite difference method. The underlying idea is that the error on the solution is of the same order as the discretization of the derivative operator. At order 2, an order 2 convergence could be expected and similarly at order 4. It is obvious that an order 4 finite difference method is easier to implement than an order 3 finite element method. Moreover, it will be easy to further increase the order of the finite difference method. 


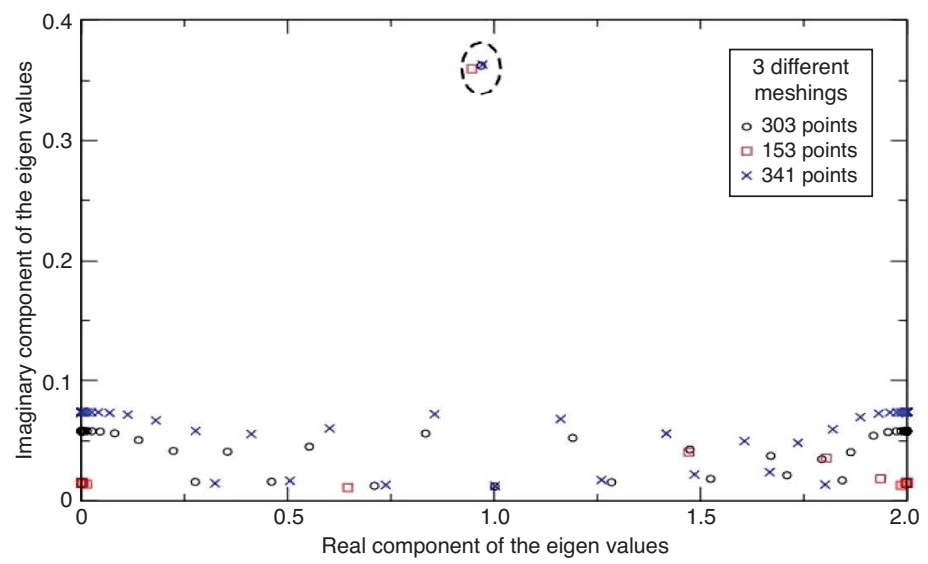

Figure 18

Set of eigen values for several different discretizations.

At each node of number $i$ of the mesh, we introduce the discrete centered scheme of order 2 or order 4 :

$$
\begin{gathered}
\varphi_{i}^{(2)}=\frac{1}{h^{2}}\left(-\frac{5}{2} \varphi_{i}+\frac{4}{3}\left(\varphi_{i-1}+\varphi_{i+1}\right)-\frac{1}{12}\left(\varphi_{i-2}+\varphi_{i+2}\right)\right) \\
\varphi_{i}^{(4)}=\frac{1}{h^{4}}\left(\begin{array}{l}
\frac{2}{8} 3 \varphi_{i}-\frac{2}{3}\left(\varphi_{i-1}+\varphi_{i+1}\right) \\
+2\left(\varphi_{i-2}+\varphi_{i+2}\right)-\frac{1}{6}\left(\varphi_{i-3}+\varphi_{i+3}\right)
\end{array}\right)
\end{gathered}
$$

in the discretized equation:

$$
\begin{aligned}
& \forall i, U_{i}\left(\varphi_{i}{ }^{\prime}-\alpha^{2} \varphi_{i}\right)-U{ }^{\prime \prime} \varphi_{i} \\
& +\frac{i \mu}{\rho \alpha}\left(\varphi_{i}^{(4)}-2 \alpha^{2} \varphi_{i}{ }^{\prime}+\alpha^{4} \varphi_{i}\right)=\frac{\beta}{\alpha}\left(\varphi_{i}{ }^{\prime}-\alpha^{2} \varphi_{i}\right)
\end{aligned}
$$

At the boundary, some complementary values have to be added; 2 values equal zero at order 2 and 3 values at order 4 .

To solve this problem of generalized eigen values and vectors, we build up a matrix and call a Lapack routine which uses Arnoldi's procedure. Calculations are carried out on a standard work station in a few minutes.

\subsection{Choice of the Eigen Values}

The number of eigen values is equal to the dimension of the linear system. But, all the eigen values are not physically meaningful and it is difficult to separate the artificial eigen values from the true values. It is necessary to sort which values have a physical sense. Therefore, we compute the same problem three times with different meshes. An elected eigen values remain at the same location through the different meshes as illustrated in Figure 18 for the eigen value (0.94, 0.36 ). An automatic procedure removes all the spurious eigen values but sometimes, a finishing touch has to be done manually.
The computed values in many cases show that the discretized eigen values converge when $h$ decreases. We enhance the final eigen value by using a Richardson's extrapolation formula from $\lambda(h / 2)$ and $\lambda(h)$.

\subsection{Results and Discussion}

We use the velocity profile displayed in Figure 6. The whole profile is on the left and there is a close-up inside the cavity on the right. The inflexion point is clearly present at the entrance of the cavity. The hydrodynamics instability is analyzed below.

In Figure 19, we compare the eigen values found by means of an FE of order 2 and FD of order 4. FE2 finds only a part of the eigen values and their values seem quite bad enough. It is clear that the P2 elements are not sufficient to correctly solve this eigen value problem. Finally, we use an FD4 method which turns out to be a good one. The most probable frequency is $1450 \mathrm{~Hz}$ with a magnitude of 3300 .



Figure 19

Comparison of eigen values according to 2 methods: FE2 and FD4. 


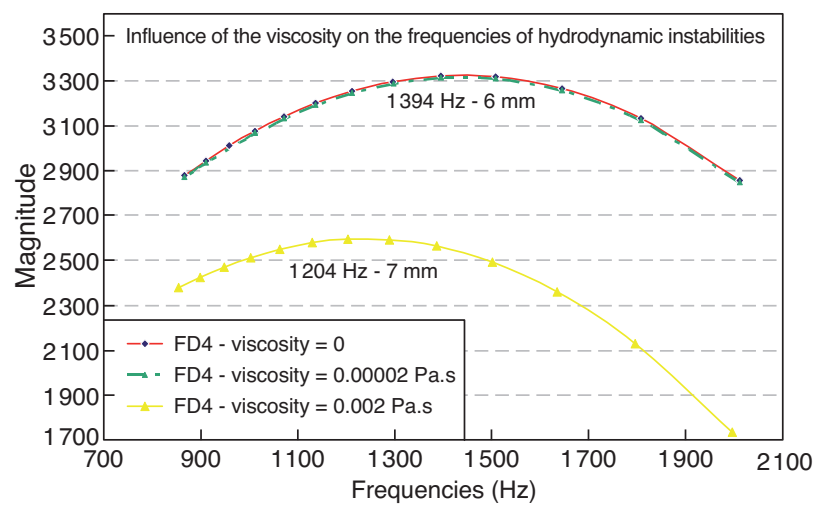

Figure 20

Comparison of FD4 solution depending on the viscosity.

Remark: increasing the order of the method leads to an increase in the size of the discretization step whilst keeping the same level of accuracy. Thus, the number of eigen values is smaller.

The influence of the viscosity is not easily accounted for. Such modeling gives a quantitative response. Figure 20 presents three models using the FD4 method:

- Rayleigh's equation (viscosity $=0$ );

- Orr-Sommerfeld equation with viscosity $=2 \cdot 10^{-5}$ Pa.s;

- Orr-Sommerfeld equation with viscosity $=2 \cdot 10^{-3} \mathrm{~Pa}$.s.

The eigen values for viscosity $=0$ and viscosity $=2 \cdot 10^{-5} \mathrm{~Pa} . \mathrm{s}$ are very close; when viscosity is 100 times greater, the OrrSommerfeld equation solution is very different according to frequency and magnitude: energy in the mixing layer is slightly dissipated by viscosity and the most probable frequency shifts from $1394 \mathrm{~Hz}$ to $1204 \mathrm{~Hz}$. Moreover, the associated wave length moves from $6 \mathrm{~mm}$ to $7 \mathrm{~mm}$. To conclude, we see that the viscosity influence is negligible for low viscosity while it plays a role, due to dissipation, for high viscosity and for high Reynolds (decreasing magnitude and frequency and increasing wave length). That is in accordance with Lin's [32] theory.

The results concerning the flow at $\bar{u}=20 \mathrm{~m} / \mathrm{s}$ are now described. The eigen values have been computed along the 8 radial lines of Figure 21. Figure 22 shows the magnitude of the eigen values depending on the frequency (Fig. $22 b$ is a close-up of the small magnitudes of Fig. 22a); their greatest magnitude (top of each curve), frequency, wave length and radial location are to be found in Table 2. For lines 1-4, we have relevant magnitudes with fairly small magnitude. Similarly for lines 8-9, phenomena belong to the main stream. Considering their radial location and their wave length, we can think they are not linked to the cavity. In any case, since their magnitude is weak, they are of little importance.

On lines 5 and 6, meaningful eigen events have to be considered. Their wave lengths correspond to the cavity length and for line 5, it can be thought that several vortices are present in the detachment zone and a traffic with several vortices exists in the shear layer. Six millimetres corresponds to the cavity length and line 6 eigen value seems to have a physical existence stimulated by the geometry of the mixing layer. To conclude, this analysis points out the presence of a $6 \mathrm{~mm}$ wave length of large amplitude at $1500 \mathrm{~Hz}$. The hydrodynamic eigen mode: $2400 \mathrm{~Hz}, 4 \mathrm{~mm}$, corresponds to a step in the $\mathrm{KH}$ instability life as the analysis of the momentum thickness indicates in Figure 12. Beyond line 7, the linear regime modelled by means of the Orr-Sommerfeld equation is probably overtaken by the not yet linear vortex rolls and touches its limits.

Orr-Sommerfeld analysis provides a tool to understand the instabilities of a flow in terms of spatial and temporal content.

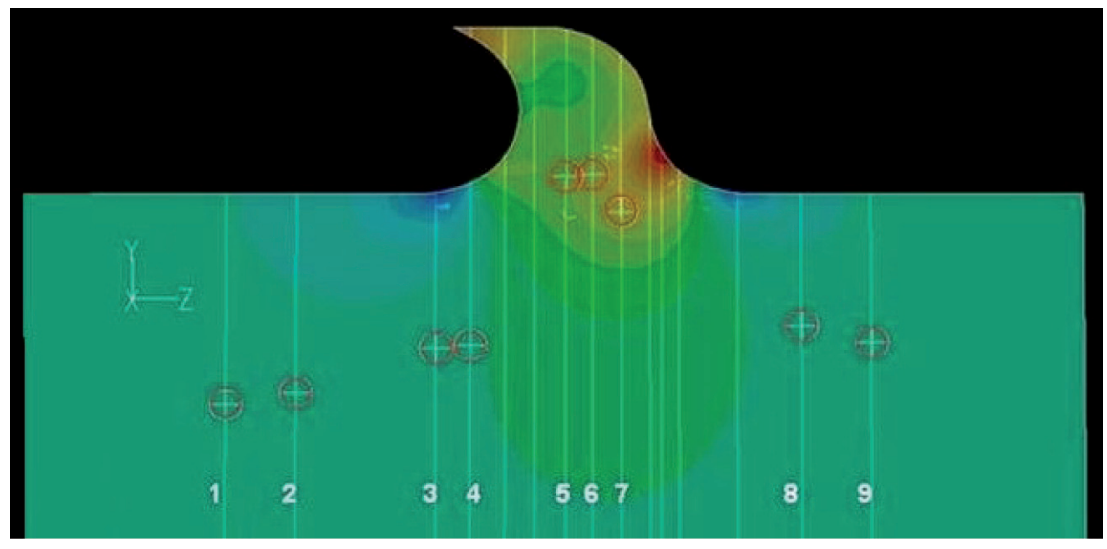

Figure 21

In an axial plane, the red circles indicate the location of the eigen functions. 

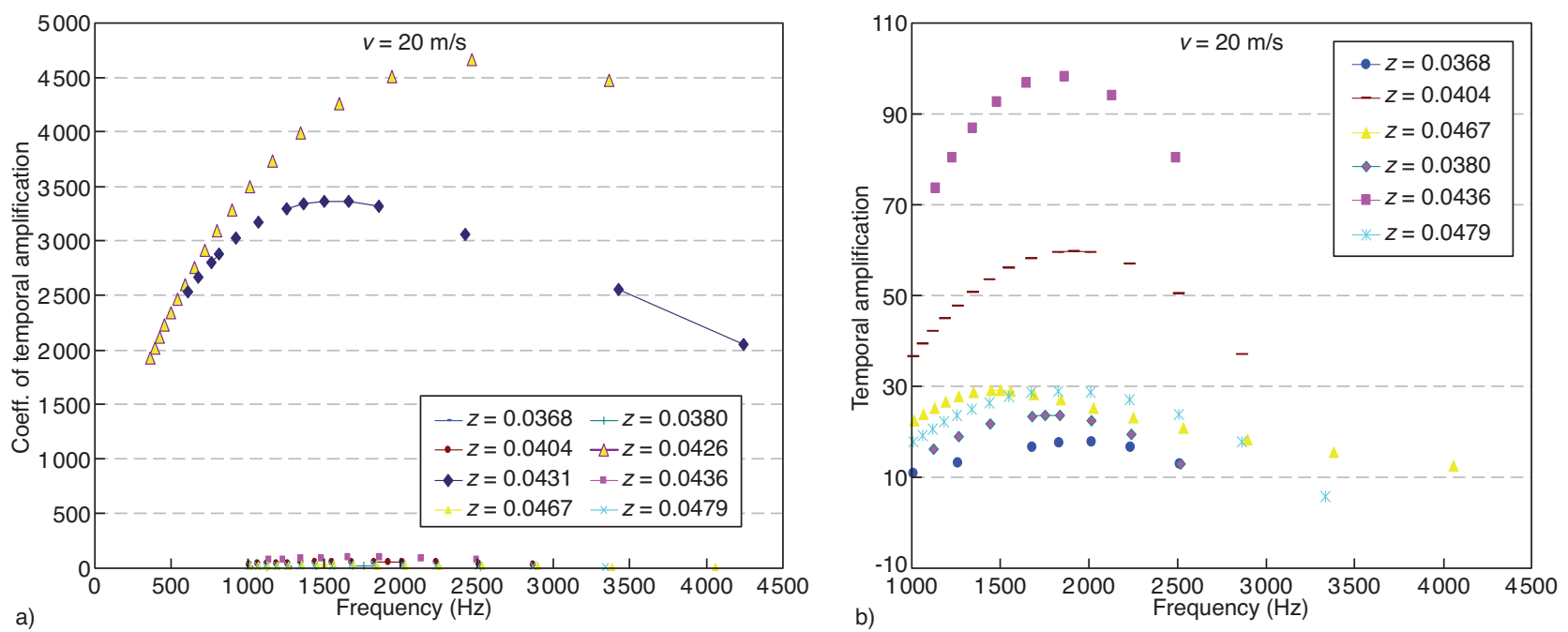

Figure 22

DF4 solution for a mean flow of $20 \mathrm{~m} / \mathrm{s}$ in a flexible hose. Close-up on the right for the smallest eigen values.

TABLE 2

The eigen values and locations of the eigen function

\begin{tabular}{c|c|c|c|c|c}
\hline $\begin{array}{c}\text { Axial } \\
\text { location }\end{array}$ & $\begin{array}{c}\text { Wave } \\
\text { length }\end{array}$ & Frequency & Magnitude & $\begin{array}{c}\text { Radial } \\
\text { location }\end{array}$ & Velocity \\
\hline No. - value & $\mathrm{mm}$ & $\mathrm{Hz}$ & & & $\mathrm{m} / \mathrm{s}$ \\
\hline $1-0.0368$ & 10.0 & 2014.6 & 17.8 & 0.03407 & 20.1 \\
\hline $2-0.0380$ & 11.5 & 1756.5 & 23.6 & 0.03430 & 20.2 \\
\hline $3-0.0404$ & 10.5 & 1915.1 & 59.7 & 0.03515 & 20.1 \\
\hline $4-0.0410$ & 6.0 & 3291.5 & 10.2 & 0.03520 & 19.7 \\
\hline $5-0.0426$ & 4.0 & 2467.3 & 4659.7 & 0.03844 & 9.9 \\
\hline $6-0.0431$ & 6.0 & 1499.3 & 3365.9 & 0.03848 & 9.0 \\
\hline $7-0.0436$ & 8.0 & 1862.1 & 98.1 & 0.03775 & 14.9 \\
\hline $8-0.0467$ & 13.5 & 1502.6 & 29.1 & 0.03557 & 20.3 \\
\hline $9-0.0479$ & 11.0 & 1827.6 & 29.0 & 0.03526 & 20.1 \\
\hline
\end{tabular}

The location of the max magnitude is plotted by a cross in a circle and written in Table 2 . A very meaningful eigen event takes place along the shear layer at a wave length equal to the cavity length.

\section{CONCLUSIONS}

The dry gas exported through a flexible riser raises a new problem of hydrodynamic instability: in the case of infinite repetition of a cavity rolled in helix around a cylindrical shape, the transverse shape ratio of the cavity is then infinite. The Reynolds number is very high $\left(\operatorname{Re}>10^{7}\right)$ and the Mach number very low $(M \sim 0.05)$. A stabilized regime with high vorticity is pointed out by means of incompressible LES modeling but the unsteady flow is unstable in the vicinity of the cavities regularly encountered. The momentum thickness between two cavities becomes constant but its high value contributes to the rise of vortices at the entrance of each cavity. In this dynamic environment, the momentum content of the vortices increases considerably all along the shear layer travel but finally increases only slightly since the shear layer length is small.

The area of vortex impacts is finely located at the output of the cavity and follows the helix geometry of the clip. Some holes judiciously calibrated at the vortex scale and set in this area could reduce the strength of the force field which hits the wall.

Indeed, at such a low Mach number ( 0.05), the fluid is nearly incompressible and acoustic phenomena of Rossitertype are unlikely to be possible. The result is no meaningful acoustic radiation but a strong solicitation of the steel structure. This result is in accordance with general considerations in the literature.

Moreover, it has been stated that radial resonance due to the cylindrical main shape of the hose was out of the frequency range of the observed in-service vibration. An important feature is that the helix cavity itself brings no relevant modification of these resonance frequencies. Consequently, a fluid-structure coupling takes place, depending on the mass and stiffness of the steel shape. Therefore, the structure enters in resonance, driving the gas column as an added mass; both structure and gas radiate same acoustic waves. LES modeling shows the cinematics of the vorticity field acting in the shear layer in front of each cavity and its link between cavities in the stabilized flow. 
The Orr-Sommerfeld equation solutions contribute to enlightening this mechanism and to locating the eigen functions the most stimulated by the instability. These solutions are cheap to carry out in terms of computer time; and the relevant eigen values can be sorted by means of several numerical solutions of different discretizations based on the finite difference method.

\section{ACKNOWLEDGMENTS}

We would like to thank Alain Coutarel who, as Technip manager, brought this problem to IFP Energies nouvelles and wholeheartedly supported its development. Thanks also to François Dupoiron, Technip project manager.

\section{REFERENCES}

1 Bamberger Y. (1997) Mécanique de l’ingénieur, solides déformables, Éd. Hermann.

2 Brac J. (2003) Propagation d'ondes acoustiques et élastiques, Lavoisier.

3 Brac J., Lanfrey P.Y., Sagaut P. (2005) Vorticity field and acoustic sources in a turbulent flow at very low Mach and high Reynolds numbers, ASME-PV\&PVC-PVP2005-71562, July, Denver.

4 Brac J., Odru P., Gerez J.M. (2002) Flow and thermal modeling in cryogenic flexible pipes, ISOPE-paper No.2002-JSC-175, KitaKyuchu.

5 Bailly C., Comte-Bellot G. (2003) Turbulence, CNRS Éditions.

6 Bailly C. (1997) Modeling of turbulent mixing noise, Lecture series-07 of Von Karman Institute, 15-18 Sept.

7 Bilanin A.J., Covert E.E. (1973) Estimation of possible excitation frequencies for shallow rectangular cavities, AIAA J. 11, 347-351.

8 Blake W.K. (1986) Mechanics of flow-induced sound and vibration, Academic Press, Vol. 1.

9 Boersma B.J. (2001) Direct numerical simulation of jet noise using different acoustic equations, Delft University of Technology, Netherlands, 14 Feb.

10 Chong M.S., Perry A.E., Cantwell B.J. (1990) A general classification of three-dimensional flow field, Phys. Fluids A 2, 765.

11 Courant R., Hilbert D. (1953) Methods of Mathematical Physics, Vol. 1, pp. 31, Interscience.

12 Drazin P.G., Howard L.N. (1966) Hydrodynamic stability of parallel flow of inviscid fluid, Elsevier, Advanced in Applied Mechanics, Vol. 9.

13 Drazin P.G., Reid W.H. (2004) Hydrodynamic stability, Cambridge University Press, 2nd Ed. Cambridge, England, pp. 69-123.

14 Ffowcs Williams J.E., Hawkings D.L. (1969) Sound generated by turbulence and surfaces in arbitrary motion, Philos. T. Roy. Soc. 264, 1151, 321-345.

15 Gharib M., Roshko A. (1987) The effect of flow oscillations on cavity drag, J. Fluid Mech. 177, 501.

16 Germain P. (1986) Mécanique, Éd. Ellipse.

17 Gloerfelt X. (2001) Bruit rayonné par un écoulement affleurant une cavité, Thèse, École Centrale de Lyon, N Ordre : 2001-26.
18 Goldstein M.E. (1976) Aeroacoustics, Mc Graw-Hill, New-York.

19 Howe M.S. (1975) Contributions to the theory of aerodynamic sound, with application to excess jet noise and the theory of the flute, J. Fluid Mech. 71, 4, 625-673.

20 Hunt J., Wray A., Moin P. (1988) Eddies, stream, and convergence zones in turbulent flows, Center of Turbulence Research Report CTR-S88, pp. 193.

21 Jeong J., Hussain F. (1995) On the identification of a vortex, J. Fluid Mech. 285, 69-94.

22 Karamcheti K. (1955) Acoustic radiation from two-dimensional rectangular cutouts in aerodynamic surfaces, NACA TN 3487.

23 Kline S.J., Reynolds W.C., Schraub F.A., Runstadler P.W. (1967) The structure of turbulent boundary layers, J. Fluid Mech. 30, 4, 741-773.

24 Larcheveque L., Sagaut P., Mary I., Labbé O. (2002) Large-eddy simulation of a compressible flow past a deep cavity, Phys. Fluids 15, 1, 193-210.

25 Larcheveque L., Sagaut P., Le T., Comte P. (2004) Large-eddy simulation of a compressible flow in a three-dimensional open cavity at high Reynolds number, J. Fluid Mech. 516, 265-301.

26 Leducq D., Perret R. (1988) Génération de vibrations par les couches limites, Société BERTIN et Cie, Société ALSTHOM, ACB et CERG, PUB 87.001.

27 Leonard A. (1980) Vortex methods for flow simulation, J. Comput. Phys. 37, 289-335.

28 Lesieur M. (1994) La turbulence, Presses universitaire de Grenoble.

29 Lighthill M.J. (1945) A new method of two-dimensional aerodynamic design, Reports and Memoranda No. 2112, April.

30 Lighthill M.J. (1952) On sound generated aerodynamically I. General theory, Proc. R. Soc. Lond. Ser. A 211.

31 Lighthill M.J. (1954) On sound generated aerodynamically II. Turbulence as a source of sound, Proc. R. Soc. Lond. Ser. A 222.

32 Lin C.C. (1944) On the stability of two-dimensional parallel flows, PNAS 30, 316-324.

33 Lin C.-C. (1955) The Theory of Hydrodynamic Stability, Cambridge Press, Cambridge, pp. 1-153.

34 Lugt H.J. (1979) The dilemma of defining a vortex, Recent Developments in Theoretical and Experimental Fluid Mechanics, Springer, pp. 309-321.

35 Michalke A. (1972) The instability of free shear layers, Elsevier, Progress in Aerospace Sciences, Vol. 12, pp. 213-216.

36 Michalke A. (1965) On spatially growing disturbances in an inviscid shear layer, J. Fluid Mech. 2, 3, 521-544.

37 Michalke A. (1964) On the inviscid instability of the hyperbolictangent velocity profile, DVL - Institut fr Turbulenzforschung, Berlin.

38 Panaitescu V.N. (2000) Une solution exacte de l'équation d'OrrSommerfeld, Bul. Inst. Polit. Iasi, t. XLVI(L), Suppliment.

39 Peters M. (1993) Aeroacoustic sources in internal flows, Thesis, Eindhoven university of technology, ISBN 90-386-0282-0, Sept.

40 Plumblee H.E., Gibson J.S., Lassiter L.W. (1962) A theoretical and experimental investigation of the acoustic response of cavities in an aerodynamic flow, U.S. Air Force Rep. WADD-TR61-75.

41 Powell A. (1964) Theory of vortex sound, J. Acoust. Soc. Am. 36, 1, 177-195.

42 Rayleigh J.W.S. (1945) Theory of sound, Tome II, 2nd ed., General Publishing Company, Vol. I and Vol. II. Dover publications. 
43 Rayleigh L. (1880) On the stability or instability of certain fluid motions, Proc. Lond. Math. Soc. 11, 57-70.

44 Ricot D. (2002) Simulation numérique d'un écoulement affleurant une cavité par la méthode Boltzmann sur réseau et application au toit ouvrant de véhicules automobiles, Thèse, École Centrale de Lyon, $\mathrm{N}^{\circ}$ Ordre : 2002-36.

45 Robinson S.K. (1991) The kinetics of turbulent boundary layer structure, PhD Dissertation, Stanford University.

46 Rockwell D. (1998) Vortex-Body Interaction, Annu. Rev. Fluid Mech. 30, 199-229.

47 Rossiter J.E. (1964) Wind tunnel experiments of the flow over rectangular cavities at subsonic and transonic speeds, Aero. Res. Counc. R. \& M., No. 3438.

48 Sarohia V. (1976) Experimental investigation of oscillations in flows over shallow cavities, paper 76-182, AIAA 14th Aerospace Sciences Meeting, Washington.
49 H. Schlichting (1979) Boundary Layer Theory, Springer, 7th Ed., Berlin, pp. 83-111.

50 Tam W., Block P. (1978) On the tones and pressure oscillations induced by flow over rectangular cavities, J. Fluid Mech. 89, 2, 373-399.

51 Tatsumi T., Gotoh K. (1959) The stability of free boundary layers between two uniform streams, J. Fluid Mech. 7, 433-441.

52 Tollmien W. (1935) Translated as, general instability criterion of laminar velocity disturbances, NACA TM-792, 1936.

53 Truesdell C. (1953) The Kinematics of Vorticity, Indiana University. 


\section{ANNEX: ORR-SOMMERFELD EQUATION}

In this annex, we state the Orr-Sommerfeld equation. First of all, we recall the incompressible steady Navier-Stokes equations:

$$
\left\{\begin{array}{c}
\nabla u .=0 \\
\frac{\partial u}{\partial t}+(u . \nabla) u=-\frac{1}{\rho} \nabla p+\frac{\mu}{\rho} \Delta u
\end{array}\right.
$$

We consider the following velocity and pressure profiles:

$$
u=\left(\begin{array}{r}
U(y)+\varepsilon u_{1}(x, y, t) \\
\varepsilon v_{1}(x, y, t)
\end{array}\right) \text { and } p=P(y)+\varepsilon p_{1}(x, y, t)
$$

The perturbed equations are deduced by introducing this velocity profile in the NS equations:

$$
\left\{\begin{array}{c}
\nabla \cdot\left(\begin{array}{c}
u_{1} \\
v_{1}
\end{array}\right)=0 \\
\varepsilon \frac{\partial}{\partial t}\left(\begin{array}{c}
u_{1} \\
v_{1}
\end{array}\right)+\left(\begin{array}{c}
\left(U+\varepsilon u_{1}\right) \frac{\partial\left(U+\varepsilon u_{1}\right)}{\partial x}+\varepsilon v_{1} \frac{\partial\left(U+\varepsilon u_{1}\right)}{\partial y} \\
\left.\left(U+\varepsilon u_{1}\right) \frac{\partial\left(\varepsilon v_{1}\right)}{\partial x}+\varepsilon v_{1} \frac{\partial\left(\varepsilon v_{1}\right)}{\partial y}\right)
\end{array}\right)=-\frac{1}{\rho} \nabla\left(P+\varepsilon p_{1}\right)+\frac{\mu}{\rho} \Delta\left(\begin{array}{c}
U+\varepsilon u_{1} \\
\varepsilon v_{1}
\end{array}\right)
\end{array}\right.
$$

We define the stream function $\Psi_{1}$ of the perturbation as:

$$
u_{1}=\frac{\partial \Psi_{1}(x, y, t)}{\partial y} \text { and } v_{1}=-\frac{\partial \Psi_{1}(x, y, t)}{\partial x}
$$

and the stream function is assumed to have the following shape:

$$
\Psi_{1}(x, y, t)=\operatorname{Re}\left(\varphi(y) e^{i(\alpha x-\beta t)}\right)
$$

$\alpha=\alpha_{r}+i \alpha_{i}$ and $\beta=\beta_{r}+i \beta_{i}$ are complex numbers, the $\alpha_{r} \in \Re$ is the spatial pulsation and $\beta_{r} \in \Re$ the temporal pulsation. The numbers $\alpha_{i} \in \Re$ and $\beta_{i} \in \Re$ are their respective expansion rates.

Temporal perturbation $\left(\alpha_{i}=0\right), \Psi_{1}(x, y, t)=e^{\beta_{i} t} \operatorname{Re}\left(\varphi(y) e^{i\left(\alpha_{r} x-\beta_{r} t\right)}\right)$

Spatial perturbation $\quad\left(\beta_{i}=0\right), \Psi_{1}(x, y, t)=e^{-\alpha_{i} x} \operatorname{Re}\left(\varphi(y) e^{i\left(\alpha_{r} x-\beta_{r} t\right)}\right)$

We deduce the real perturbed fields:

$$
\left(\begin{array}{l}
u_{1} \\
v_{1}
\end{array}\right)=\left(\begin{array}{c}
\operatorname{Re}\left(\varphi^{\prime}(y) e^{i(\alpha x-\beta t)}\right) \\
\operatorname{Re}\left(-i \alpha \varphi(y) e^{i(\alpha x-\beta t)}\right)
\end{array}\right) \text { and } p_{1}(x, y, t)=\operatorname{Re}\left(p(y) e^{-i(\alpha x-\beta t)}\right)
$$

In order to linearize this equation, we neglect the term in $\varepsilon$ and we obtain:

$$
\left(\begin{array}{c}
-i \beta \varphi^{\prime} \\
-\alpha \beta \varphi
\end{array}\right)+\left(\begin{array}{c}
i \alpha U \varphi^{\prime}-i \alpha \varphi U^{\prime} \\
-(i \alpha)^{2} U \varphi
\end{array}\right)=-\frac{1}{\rho}\left(\begin{array}{c}
i \alpha p \\
p^{\prime}
\end{array}\right)+\frac{\mu}{\rho}\left(\begin{array}{c}
\varphi^{\prime \prime \prime}-\alpha^{2} \varphi^{\prime} \\
-i \alpha\left(\varphi^{\prime \prime}-\alpha^{2} \varphi\right)
\end{array}\right)
$$

Derivating the first line of this equation and introducing it in the second line, we obtain the Orr-Sommerfeld equation:

$$
\left(U-\frac{\beta}{\alpha}\right)\left(\varphi "-\alpha^{2} \varphi\right)-U " \varphi=-\frac{i \mu}{\alpha \rho}\left(\varphi^{(4)}-2 \alpha^{2} \varphi^{\prime \prime}+\alpha^{4} \varphi\right)
$$




\section{Dimensionless equation}

Some characteristic sizes are chosen: length $L$, and velocity $U_{m}$. We deduce the following dimensionless variables:

$$
\bar{y}=\frac{y}{L}, \bar{u}=\frac{u}{U_{m}}, \bar{\nabla}=L \nabla, \bar{\varphi}(y)=\frac{\varphi(y)}{U_{m} L}
$$

The Orr-Sommerfeld equation becomes:

$$
\left(U-\frac{\beta L}{U_{m}} \frac{1}{\alpha L}\right)\left(\varphi "-(\alpha L)^{2} \varphi\right)-U^{\prime \prime} \varphi=-i \frac{\mu}{\rho U_{m}} \frac{1}{\alpha L}\left(\varphi^{(4)}-2(\alpha L)^{2} \varphi^{\prime \prime}+(\alpha L)^{4} \varphi\right)
$$

Three dimensionless numbers appear:

- Reynolds number $\operatorname{Re}=\frac{\rho U_{m} L}{\mu}$

- Strouhal number $S t=\frac{f L}{U_{m}}=\frac{1}{2 \pi} \frac{\beta L}{U_{m}}$ number $\alpha L$, real when $\alpha$ is real. 